\title{
On Gradings Modulo 2 of Simple Lie Algebras in Characteristic 2
}

\author{
Andrey KRUTOV ${ }^{\dagger \ddagger}$ and Alexei LEBEDEV $\S$ \\ $\dagger$ Institute of Mathematics, Polish Academy of Sciences, \\ ul. Śniadeckich 8, 00-656 Warszawa, Poland \\ ¥ Independent University of Moscow, Bolshoi Vlasyevskij Pereulok 11, 119002, Moscow, Russia \\ E-mail:a.o.krutov@gmail.com \\ $\S$ Equa Simulation AB, Stockholm, Sweden \\ E-mail: alexeylalexeyl@mail.ru
}

Received January 10, 2018, in final form November 30, 2018; Published online December 10, 2018 https://doi.org/10.3842/SIGMA.2018.130

\begin{abstract}
The ground field in the text is of characteristic 2. The classification of modulo 2 gradings of simple Lie algebras is vital for the classification of simple finitedimensional Lie superalgebras: with each grading, a simple Lie superalgebra is associated, see arXiv:1407.1695. No classification of gradings was known for any type of simple Lie algebras, bar restricted Jacobson-Witt algebras (i.e., the first derived of the Lie algebras of vector fields with truncated polynomials as coefficients) on not less than 3 indeterminates. Here we completely describe gradings modulo 2 for several series of Lie algebras and their simple relatives: of special linear series, its projectivizations, and projectivizations of the derived Lie algebras of two inequivalent orthogonal series (except for $\mathfrak{o}_{\Pi}(8)$ ). The classification of gradings is new, but all of the corresponding superizations are known. For the simple derived Zassenhaus algebras of height $n>1$, there is an $(n-2)$-parametric family of modulo 2 gradings; all but one of the corresponding simple Lie superalgebras are new. Our classification also proves non-triviality of a deformation of a simple $3 \mid 2$-dimensional Lie superalgebra (new result).
\end{abstract}

Key words: modular vectorial Lie algebra; characteristic 2; simple Lie algebra; simple Lie superalgebra

2010 Mathematics Subject Classification: 17B50; 17B20; 17B70

\section{Introduction}

\section{$1.1 \quad$ Basic definitions}

Hereafter $\mathbb{K}$ is an algebraically closed field of characteristic $p=2$, unless otherwise specified; all algebras are finite-dimensional; for a review of simple vectorial Lie (super)algebras over $\mathbb{K}$ and basic background, see [3].

Lie superlagebras in characteristic 2. A Lie superalgebra is a superspace $\mathfrak{g}=\mathfrak{g}_{\overline{0}} \oplus \mathfrak{g}_{\overline{1}}$ such that the even part $\mathfrak{g}_{\overline{0}}$ is a Lie algebra, the odd part $\mathfrak{g}_{\overline{1}}$ is a $\mathfrak{g}_{\overline{0}}$-module, and on $\mathfrak{g}_{\overline{1}}$, a squaring (roughly speaking, the halved bracket) is defined as a map

$$
\begin{aligned}
& x \mapsto x^{2} \quad \text { such that }(a x)^{2}=a^{2} x^{2} \text { for any } x \in \mathfrak{g}_{\overline{1}} \text { and } a \in \mathbb{K}, \text { and } \\
& (x+y)^{2}-x^{2}-y^{2} \text { is a bilinear form on } \mathfrak{g}_{\overline{1}} \text { with values in } \mathfrak{g}_{\overline{0}} .
\end{aligned}
$$

Then the bracket of odd elements is defined to be

$$
[x, y]:=(x+y)^{2}-x^{2}-y^{2} .
$$


The Jacobi identity involving odd elements takes the following form:

$$
\left[x^{2}, y\right]=[x,[x, y]] \quad \text { for any } x \in \mathfrak{g}_{\overline{1}}, y \in \mathfrak{g}_{\overline{0}}, \quad\left[x^{2}, x\right]=0 \quad \text { for any } x \in \mathfrak{g}_{\overline{1}} .
$$

Divided powers. There are two natural integer bases of the commutative algebra $\mathbb{C}[x]$ of polynomials in $m$ indeterminates $x=\left(x_{1}, \ldots, x_{m}\right)$ : the monomial one and the basis of divided powers, constructed as follows. For any multi-index $\underline{r}=\left(r_{1}, \ldots, r_{m}\right)$, where $r_{1}, \ldots, r_{m}$ are non-negative integers, we set

$$
u_{i}^{\left(r_{i}\right)}:=\frac{x_{i}^{r_{i}}}{r_{i} !} \quad \text { and } \quad u^{(\underline{r})}:=\prod_{1 \leq i \leq m} u_{i}^{\left(r_{i}\right)} .
$$

These $u^{(\underline{r})}$ form an integer basis of $\mathbb{C}[x]$. Their multiplication relations are

$$
u^{(\underline{r})} \cdot u^{(\underline{s})}=\left(\begin{array}{c}
\underline{r}+\underline{s} \\
\underline{r}
\end{array}\right) u^{(\underline{r}+\underline{s})}, \quad \text { where } \quad\left(\begin{array}{c}
\underline{r}+\underline{s} \\
\underline{r}
\end{array}\right)=\prod_{1 \leq i \leq m}\left(\begin{array}{c}
r_{i}+s_{i} \\
r_{i}
\end{array}\right) .
$$

Being interested in simple Lie algebras, observe that every simple $\mathbb{Z}$-graded Lie algebra $\mathfrak{g}=\oplus \mathfrak{g}_{i}$ of vector fields is transitive, i.e., such that

$$
\left[\mathfrak{g}_{\leq 0}, x\right]=0 \text { for a given } x \in \mathfrak{g}_{>0} \text { implies } x=0 \text {, where } \mathfrak{g}_{\leq 0}=\oplus_{i \leq 0} \mathfrak{g}_{i}, \mathfrak{g}_{>0}=\oplus_{i>0} \mathfrak{g}_{i} .
$$

For an arbitrary field $\mathbb{K}$ of characteristic $p>0$, it is easy to see that the Lie algebra $\mathfrak{d e r} \mathbb{K}[x]$ is not transitive in the $\mathbb{Z}$-grading induced by the standard $\mathbb{Z}$-grading of $\mathbb{K}[x]$, i.e., $\operatorname{deg} x_{i}=1$ for all $i$. The situation is remedied if we consider the commutative algebra $\mathbb{K}[u]$ spanned by all the elements $u^{(\underline{r})}$ with multiplication relations (1.1). For any $m$-tuple $\underline{N}=\left(N_{1}, \ldots, N_{m}\right)$, where $N_{i}$ are either positive integers, or infinity, denote (we set $p^{\infty}:=\infty$ )

$$
\mathcal{O}(m ; \underline{N}):=\mathbb{K}[u ; \underline{N}]=\operatorname{Span}_{\mathbb{K}}\left(u^{(\underline{r})} \mid r_{i}<p^{N_{i}}\right) .
$$

The algebra $\mathbb{K}[u]$ and its subalgebras $\mathbb{K}[u ; \underline{N}]$ are called the algebras of divided powers; they are analogs of the polynomial algebra. Let $\mathbb{1}:=(1, \ldots, 1)$ denote the shearing vector $\underline{N}$ with the smallest values of heights $N_{i}$ of the indeterminates.

Clearly, if $N_{i} \neq 1$ for at least one $i$, then $\mathcal{O}(m ; \underline{N})$ has more than $m$ generators: namely $y_{i, j}:=u_{i}^{\left(p^{j-1}\right)}$. Any derivation $D$ of a given algebra is determined by the values of $D$ on the generators, so $\mathfrak{d e r} \mathcal{O}(m ; \underline{N})$ has more than $m$ analogs of partial derivations: one for each generator, with a functional parameter $f_{i j}(u) \in \mathcal{O}(m ; \underline{N})$ - coefficient of each partial derivative $\partial_{y_{i, j}}$, whereas the coefficients of $\partial_{y_{i, j}} \in \mathfrak{v e c t}\left(\sum N_{i} ; \mathbb{1}\right)$ belong to $\mathcal{O}\left(\sum N_{i} ; \mathbb{1}\right)$. Following the definitions over $\mathbb{C}$ we are interested in subalgebras of $\mathfrak{d e r} \mathcal{O}(m ; \underline{N})$ such that the dimension of the nonpositive part (in the standard $\mathbb{Z}$-grading) is equal to $m$.

Solution was found long ago: one has to introduce distinguished partial derivatives $\partial_{i}$, each of them serving as several partial derivatives (corresponding to $u_{i}, u_{i}^{(p)}, u_{i}^{\left(p^{2}\right)}, \ldots$ ) at once (if instead of the usual powers we use divided ones, the sign $(-1)^{j-1}$ is not needed):

$$
\partial_{i}\left(u_{j}^{(k)}\right):=\delta_{i j} u_{j}^{(k-1)} \quad \text { for all } k \text {, i.e., } \partial_{i}=\sum_{j \geq 1}(-1)^{j-1} y_{i, 1}^{p-1} \cdots y_{i, j-1}^{p-1} \partial_{y_{i, j}} .
$$

The general vectorial Lie algebra of distinguished derivations ${ }^{1}$

$$
\mathfrak{v e c t}(m ; \underline{N})=\operatorname{Span}_{\mathbb{K}}\left(f \partial_{k} \mid f \in \mathcal{O}(m ; \underline{N}), k=1, \ldots, m\right)
$$

is simple (except for $m=1$ and $p=2$ ).

\footnotetext{
${ }^{1}$ For $p>0$, the Lie algebra $\mathfrak{v e c t}(m ; \underline{N})$ is called Jacobson-Witt algebra if $\underline{N} \neq \mathbb{1} ;$ it is usually denoted $W(m ; \underline{N})$ for any $m$ and $\underline{N}$; if $m=1$, it is called Zassenhaus algebra. Jacobson-Witt algebras are simple if $m>1$; there is no special name for the simple derived $\mathfrak{v e c t}^{(1)}(1 ; \underline{n})$, see a discussion in [14]. The Lie algebra of divergence-free, or "special", vector fields is denoted $\mathfrak{s v e c t}(m ; \underline{N})$, usually abbreviated to $S(m ; \underline{N})$.
} 
$\boldsymbol{p}$-structure. If $\mathfrak{g}$ is a Lie algebra, then for every $x \in \mathfrak{g}$, the operator $\left(\operatorname{ad}_{x}\right)^{p}$ is a derivation of $\mathfrak{g}$. If this derivation is inner for every $x \in \mathfrak{g}$, then the Lie algebra $\mathfrak{g}$ is said to be restricted or having a $p$-structure. More specifically, a $p$-structure on $\mathfrak{g}$ is a map $[p]: \mathfrak{g} \rightarrow \mathfrak{g}, x \mapsto x^{[p]}$ such that

$$
\begin{aligned}
& {\left[x^{[p]}, y\right]=\left(\operatorname{ad}_{x}\right)^{p}(y) \quad \text { for any } x, y \in \mathfrak{g},} \\
& (a x)^{[p]}=a^{p} x^{[p]} \quad \text { for any } a \in \mathbb{K}, x \in \mathfrak{g}, \\
& (x+y)^{[p]}=x^{[p]}+y^{[p]}+\sum_{1 \leq i \leq p-1} s_{i}(x, y) \quad \text { for any } x, y \in \mathfrak{g},
\end{aligned}
$$

where $s_{i}(x, y)$ is the coefficient of $\lambda^{i-1}$ in $\left(\operatorname{ad}_{\lambda x+y}\right)^{p-1}(x)$.

\section{Remark 1.1.}

1) If the Lie algebra $\mathfrak{g}$ is without center, then the last two conditions of (1.3) follow from the first one. There might be more than one $p$-structure on one Lie algebra; all of them are equal modulo center. Hence, on any simple Lie algebra, there is at most one $p$-structure.

2) The following condition is sufficient for a Lie algebra $\mathfrak{g}$ to possess a $p$-structure: for a basis $\left\{g_{i}\right\}_{i \in I}$ of $\mathfrak{g}$, there exist elements $g_{i}^{[p]}$ such that

$$
\left[g_{i}^{[p]}, y\right]=\left(\operatorname{ad}_{g_{i}}\right)^{p}(y) \quad \text { for any } \quad y \in \mathfrak{g} \text {. }
$$

We consider the following problem.

Problem 1.2. For any finitely generated commutative group $G$, classify $G$-gradings of simple finite-dimensional Lie algebras over $\mathbb{K}$.

Lie algebras for which a solution of Problem 1.2 is known. Although we are interested in a particular case of Problem 1.2, let us briefly review the known general results.

- For $p=0$, for a clear exposition of the solution (for the $\mathbb{Z}$ - and $\mathbb{Z} / n$-gradings), see the book [17, around p. 500], and also [13].

- For $p \neq 2,[18]$ is a very lucid review of the cases where the solution of Problem 1.2 has been found; for further details, see [1, 19]; for examples of applications of certain $G$-gradings, see $[20]$.

- For $p=2$, the result of [1] is as follows: all $G$-gradings of the Jacobson-Witt algebra $W(m ; \mathbb{1})$ (it is $\operatorname{vect}(m ; \mathbb{1}$ ) in our notation) for $m \geq 3$ are given by $G$-gradings of the corresponding algebra of divided powers $\mathcal{O}(m ; \mathbb{1})$ due to an isomorphism of their automorphism group schemes [27]. The classification of such gradings is given. Any $G$-grading of $\mathcal{O}(m ; \mathbb{1})$ is equivalent, up to an algebra automorphism, to one that can be described as follows. For a given $s$ such that $0 \leq s \leq m$, and $a_{1}, \ldots, a_{m} \in G$, set

$$
\mathcal{O}_{g}=\operatorname{Span}\left\{\left(1+x_{1}\right)^{j_{1}} \cdots\left(1+x_{s}\right)^{j_{s}} x_{s+1}^{j_{s+1}} \cdots x_{m}^{j_{m}} \mid j_{i}=0 \text { or } 1, \sum_{1 \leq i \leq m} j_{i} a_{i}=g\right\},
$$

where $a_{1}, \ldots, a_{m} \in G$ are the respective degrees of the indeterminates generating $\mathcal{O}(m ; \mathbb{1})$, i.e.,

$$
1+x_{1}, \ldots, 1+x_{s}, x_{s+1}, \ldots, x_{m} .
$$

For $p>0$ and the restricted Lie algebras considered in [1] (for $p=2$ : Jacobson-Witt alge$\operatorname{bras} \operatorname{vect}(m ; \underline{N})$ for $m \geq 3$; for $p>2$ : Jacobson-Witt algebras, simple relatives of Hamiltonian algebras and algebras of divergence-free vector fields), the only possible $\mathbb{Z} / 2$-gradings are generated by those described in equation (1.4). For non-restricted Lie algebras, we know several examples of $\mathbb{Z} / 2$-gradings of Kaplansky algebras, see [8]. 


\subsection{Problem 1.2 for $G=\mathbb{Z} / 2$ and $p=2$ : an application of the solution}

In [7], there are offered two methods for constructing a simple finite-dimensional Lie superalgebra from every simple finite-dimensional Lie algebra over a field of characteristic 2 ; it is proved that every simple finite-dimensional Lie superalgebra can be obtained by one of these two methods (queerification and "method 2"). The "method 2" depends on the $\mathbb{Z} / 2$-gradings of the simple Lie algebra which is being superized. Let us recall the method:

Let $\mathfrak{g}=\mathfrak{g}_{\overline{0}} \oplus \mathfrak{g}_{\overline{1}}$ be a simple Lie algebra with a $\mathbb{Z} / 2$-grading gr. Let the 1 -step restricted closure of $\mathfrak{g}$ associated with the grading gr be

$$
\begin{aligned}
\mathfrak{g}^{\langle 1\rangle}:= & \text { the minimal Lie subalgebra of the restricted closure } \overline{\mathfrak{g}} \text { containing } \mathfrak{g} \\
& \text { and all the elements } x^{[2]} \text {, where } x \in \mathfrak{g}_{\overline{1}} .
\end{aligned}
$$

Clearly, there is a single way to extend the grading gr from $\mathfrak{g}$ to $\mathfrak{g}^{\langle 1\rangle}$; we assume this extension performed. On the space of $\mathfrak{g}^{\langle 1\rangle}$, define the structure of a Lie superalgebra denoted (in what follows, we often omit indicating the grading gr since it enters the definition of $\mathfrak{g}^{\langle 1\rangle}$ )

$$
\mathfrak{s}\left(\mathfrak{g}^{\langle 1\rangle}, \mathrm{gr}\right) \quad \text { by setting } x^{2}:=x^{[2]} \text { for any } x \in \mathfrak{g}_{\overline{1}},
$$

and retaining the bracket of any even element with any other element. The Lie superalgebra $\mathfrak{s}\left(\mathfrak{g}^{\langle 1\rangle}\right)$ is simple, see [7]. Our strategic goal is classification of simple Lie (super)algebras, so we formulate our description of $\mathbb{Z} / 2$-gradings gr of $\mathfrak{g}$ in terms of superizations $\mathfrak{s}\left(\mathfrak{g}^{\langle 1\rangle}\right)$ whenever we can.

\subsection{How we seek $\mathbb{Z} / 2$-gradings}

Let $\mathfrak{g}$ be a Lie algebra, and let $\mathfrak{g}=\mathfrak{g}_{\overline{0}} \oplus \mathfrak{g}_{\overline{1}}$ be its $\mathbb{Z} / 2$-grading. For an any $x \in \mathfrak{g}$, we denote by $x_{\overline{0}}$ its even part and by $x_{\overline{1}}$ its odd part. We have

$$
[x, y]=\left[x_{\overline{0}}+x_{\overline{1}}, y_{\overline{0}}+y_{\overline{1}}\right]=\left[x_{\overline{0}}, y_{\overline{0}}\right]+\left[x_{\overline{1}}, y_{\overline{1}}\right]+\left[x_{\overline{0}}, y_{\overline{1}}\right]+\left[x_{\overline{1}}, y_{\overline{0}}\right] \quad \text { for any } x, y \in \mathfrak{g} .
$$

This implies

$$
([x, y])_{\overline{0}}=\left[x_{\overline{0}}, y_{\overline{0}}\right]+\left[x_{\overline{1}}, y_{\overline{1}}\right] \quad \text { and } \quad([x, y])_{\overline{1}}=\left[x_{\overline{0}}, y_{\overline{1}}\right]+\left[x_{\overline{1}}, y_{\overline{0}}\right] .
$$

Finally, let $U \in \operatorname{End}(\mathfrak{g})$, where $\mathfrak{g}$ is considered as a vector space, be the projection to the $\mathfrak{g}_{1}$, i.e., $U x=x_{\overline{1}}$ and $(I-U) x=x_{\overline{0}}$, where $I$ is the identity operator. A linear operator $U$ is such a projection to the odd part of $\mathfrak{g}$ in some $\mathbb{Z} / 2$-grading if and only if it satisfies the following conditions for all $x, y \in \mathfrak{g}$ :

$$
\begin{aligned}
& U^{2}=U, \\
& U[x, y]=[U x,(I-U) y]+[(I-U) x, U y]=-2[U x, U y]+[U x, y]+[x, U y] .
\end{aligned}
$$

Having fixed a basis in $\mathfrak{g}$, we express these conditions in terms of structure constants $c_{k}^{i j}$ as (summation over repeated indices is assumed)

$$
\begin{aligned}
U_{j}^{k} U_{k}^{i} & =U_{j}^{i}, \\
c_{l}^{i j} U_{k}^{l} & =c_{k}^{i l} U_{l}^{j}+c_{k}^{l j} U_{l}^{i}-2 c_{k}^{l m} U_{l}^{i} U_{m}^{j} .
\end{aligned}
$$

For $p=2$, equation (1.8b) becomes linear and easy (for example, for Mathematica-based computer package SuperLie, see [15]) to solve. There remains, however, a problem to be solved:

condition (1.8a) is still quadratic,

we need equivalence classes of solutions $U \bmod \operatorname{Aut}(\mathfrak{g})$, not individual operators. 
A comment: gradings and derivations. For a general discussion of relation between gradings and derivations, and interesting examples for $p=2$, see [8]. For $p=3$, an example illustrating the said discussion is the $\mathbb{Z} / 4$-grading of the Skryabin algebra by, see [16].

The Lie algebra $\mathfrak{d e r} \mathfrak{v e c t}(m ; \underline{N})$ of all derivations of $\mathfrak{v e c t}(m ; \underline{N})$ coincides with the $p$-envelope of $\mathfrak{v e c t}(m ; \underline{N})$. So the problem of classification of equivalence classes of $\mathbb{Z} / p$-gradings of $\mathfrak{v e c t}(m ; \underline{N})$ reduces to the classification of equivalence classes of toral elements in $\operatorname{der} \mathfrak{v e c t}(m ; \underline{N})$. The equivalence classes of maximal tori with respect to the group of automorphisms are described in $[21,29]$, and the answer depends on $N_{1}-2$ parameters for $m=1$.

\subsection{Our results}

1) We classify the $\mathbb{Z} / 2$-gradings of the Lie algebras of series $\mathfrak{s l}$, their simple subquotients, and simple derived or subquotients of both orthogonal series $-\mathfrak{o}_{I}$ and $\mathfrak{o}_{\Pi},-$ except for $\mathfrak{o}_{\Pi}(8)$. In all the cases considered, these gradings yield the known superizations of the corresponding Lie algebras. Note that one of the gradings of $\mathfrak{o}^{(1)}(3)$ has no analogs among the $\mathbb{Z} / 2$ gradings of the simple 3 -dimensional Lie algebras for $p \neq 2$. This unusual grading has analogs among $\mathbb{Z} / 2$-gradings of $\mathfrak{v e c t}^{(1)}(1 ; \underline{n})$, of which $\mathfrak{o}^{(1)}(3) \simeq \mathfrak{v e c t}^{(1)}(1 ; \underline{2})$ is a particular case.

2) The classification of $\mathbb{Z} / 2$-gradings of $\mathfrak{o}^{(1)}(3)$ has one more application: it gives the shortest known proof of the fact that the deform of $\mathfrak{o o}_{I \Pi}^{(1)}(1 \mid 2)$, one of the two superizations of $\mathfrak{o}^{(1)}(3)$, found in [5], is a "true" one, not "semitrivial", see Remark 6.6; this fact is new and unexpected (although the deform itself, $\mathfrak{o o}_{I I}^{(1)}(1 \mid 2)$, is known).

3 ) We describe an $(n-2)$-parametric collection of $\mathbb{Z} / 2$-gradings of $\mathfrak{v e c t}^{(1)}(1 ; \underline{n})$. For $n=2$, these gradings yield three non-isomorphic Lie superalgebras.

For $n>2$, these $\mathbb{Z} / 2$-grading yield

a) purely even Lie superalgebra $\mathfrak{v e c t}^{(1)}(1 ; \underline{n} \mid 0)$;

b) an $(n-2)$-parametric family of filtered deforms of $\mathfrak{k}(1 ; \underline{n-1} \mid 1)$;

c) a filtered deform of $\mathfrak{q}(\mathfrak{v e c t}(1 ; \underline{n-1}))$.

Examples where $\mathbb{Z} / 2$-gradings of "the same algebra" for $p \neq 2$ and $p=2$ differ or where previously unknown $\mathbb{Z} / 2$-gradings have been found. These are the most interesting of our results.

1) It is known that for any $p$, there is only one simple Lie algebra of dimension 3: for $p \neq 2$, it is $\mathfrak{o}(3) \simeq \mathfrak{s l}(2)$; for $p=2$, it is $\mathfrak{o}^{(1)}(3)$, the derived of $\mathfrak{o}(3)$.

For $p \neq 2$, it is also known that there is only one (as always, up to an automorphism) nontrivial $\mathbb{Z} / 2$-grading, and hence if the superization procedure had been defined for $p \neq 2$, the superization of $\mathfrak{s l}(2)$ would have been unique, $\mathfrak{s l}(1 \mid 1)$.

For $p=2$, there are 2 inequivalent nontrivial $\mathbb{Z} / 2$-gradings of $\mathfrak{o}^{(1)}(3)$, as we will see below. The corresponding non-isomorphic, see [22], Lie superalgebras are $\mathfrak{o o}_{I \Pi}^{(1)}(1 \mid 2)$ and $\mathfrak{o o}_{I I}^{(1)}(1 \mid 2)$. (It is an interesting open problem to find out if the Lie superalgebra $\mathfrak{o v}_{I I}^{(1)}(1 \mid 2 n)$ is a deform of $\mathfrak{o o}_{I \Pi}^{(1)}(1 \mid 2 n)$ for $n>1$; this requires to consider $\mathbb{Z} / 2$-gradings and deforms of $\mathfrak{k}(2 n+1 ; \mathbb{1})$ for $n>0$.)

2) For $p \neq 2$, the $\mathbb{Z} / 2$-gradings of the Zassenhaus algebra $\mathfrak{v e c t}(1 ; \underline{n})$ were only known for $n=1$, i.e., for the restricted algebras, see [1] (and, for $n>1$, obvious $\mathbb{Z} / 2$-gradings with some of either $x$ or $x+1$ declared odd). We have found new $(n-2)$-parametric family of $\mathbb{Z} / 2$-gradings in the non-restricted case. These gradings yield superizations which have no known analogs in the case $p \neq 2$. 
Remark 1.3. Describing $\mathfrak{d e r} \mathfrak{g}$ for various Lie algebras $\mathfrak{g}$, we heavily rely on Permyakov's results [23] used in [2]. Consider, for example, $\mathfrak{s l}(2 n)$ for $n>1$. Actually, Permyakov [23] does not explicitly describe $\mathfrak{d e r} \mathfrak{s l}(2 n)$, he just shows that $\operatorname{dim} H^{1}(\mathfrak{s l}(2 n), \mathfrak{s l}(2 n))=1$, this is the codimension of the subalgebra of inner derivations in $\mathfrak{d e r} \mathfrak{s l}(2 n)$. However, it is easy to see that every element of $\mathfrak{p g l}(2 n)$ described here represents a derivation of $\mathfrak{s l}(2 n)$; different elements represent different derivations; every inner derivation is represented by some element, and the codimension of the subalgebra or inner derivations of $\mathfrak{s l}(2 n)$ is equal to 1 ; therefore, $\mathfrak{p g l}(2 n)$ is isomorphic to $\mathfrak{d e r} \mathfrak{s l}(2 n)$. Similar considerations apply when we refer to [2, 23] while describing algebras of derivations of other Lie algebras.

\subsection{Open questions}

For $G=\mathbb{Z} / 2$, the gradings (1.4) with $s=0$ of $\mathfrak{v e c t}(m ; \mathbb{1})$ for $m>2$ yield $\mathfrak{v e c t}(k ; \mathbb{1} \mid m-k)$, where the vectors $\mathbb{1}$ have $m$ and $k$ coordinates, respectively. These are known superizations. If $s \neq 0$ in (1.4) and $a_{i}=\overline{1}$ for some of the indices $i \leq s$, then the corresponding indeterminates $1+x_{i}$ are odd; at the moment we are unable to identify the resulting Lie superalgebra.

The list of simple Lie algebras for which Problem 1.2 is open:

- For $p>3$, the simple vectorial Lie algebras for the shearing vector $\underline{N} \neq \mathbb{1}$, and the deforms (results of deformations) thereof (for their classification and description, see [20, 25, 28]).

- For $p=3$, the simple vectorial Lie algebras; in particular, exceptional ones, mainly discovered by Skryabin and lucidly described in [16], and their deforms to be described (for a review, see [5]); the deforms of $\mathfrak{o}(5)$ and Brown algebras described in [9].

- For $p=2$, there are many examples of simple Lie algebras of types not existing for $p \neq 2$, see $[2,3,4,8,12,14,26]$, and $[5,6]$.

- We also have to consider the simple relative of $\mathfrak{o}_{\Pi}(8)$.

Conjecture 1.4 (D. Leites). For (the simple derived of) Lie algebras with indecomposable Cartan matrix not considered in this note, i.e., parametric families of Weisfeiler-Kac algebras, all their $\mathbb{Z} / 2$-gradings correspond, conjecturally, to their known superizations listed in [6].

\section{The $\mathfrak{s l}(n)$ and $\mathfrak{p s l}(2 n)$ for $n>2$ : same answer as for $p \neq 2$}

Clearly, $\mathfrak{s l}(0 \mid n)=\mathfrak{s l}(n)$ and $\mathfrak{p s l}(0 \mid 2 n)=\mathfrak{p s l}(2 n)$.

Theorem 2.1. All $\mathbb{Z} / 2$-gradings of the Lie algebras $\mathfrak{s l}(n)$ for $n>2$ and $\mathfrak{p s l}(2 n)$ for $n>2$ are analogous to those for $p=0$, i.e., correspond to $\mathfrak{s l}(k \mid n-k)$ for $k=0, \ldots,\left[\frac{n}{2}\right]$ and $\mathfrak{p s l}(k \mid 2 n-k)$ for $k=0, \ldots, n$.

Remark 2.2. The algebra $\mathfrak{p s l}(2)$ is not simple, so we do not consider algebras $\mathfrak{s l}(2)$ and $\mathfrak{p s l}(2)$. We do not consider the algebra $\mathfrak{p s l}(4)$ in this section either, since its algebra of derivations is more complicated than the algebra of derivations of $\mathfrak{p s l}(2 n)$ for $n>2$; however, $\mathfrak{p} \mathfrak{s l}(4)$ is isomorphic to $\mathfrak{o}_{\Pi}^{(2)}(6)$, and the answer in this case is given in Remark 5.2.

Proof. The $\mathfrak{s l}(2 n+1)$ series $(n>0)$. All derivations of $\mathfrak{s l}(2 n+1)$ are inner ones, see [23], so the general solution of the linear equation (1.7b) is $U=\operatorname{ad}_{A}$, where $A \in \mathfrak{s l}(2 n+1)$. The nonlinear equation (1.7a) reduces then to $\operatorname{ad}_{A}=\left(\operatorname{ad}_{A}\right)^{2}$. Now recall that there is the 2-structure on $\mathfrak{s l}(2 n+1)$ given by $A^{[2]}=A^{2}$, see $[6,7]$. Therefore

$$
\operatorname{ad}_{A}=\left(\operatorname{ad}_{A}\right)^{2}=\operatorname{ad}_{A^{[2]}}=\operatorname{ad}_{A^{2}},
$$


and since $\mathfrak{s l}(2 n+1)$ has no center, we have $A=A^{2}$, i.e., $A$ is a projection. Since $\operatorname{tr} A$ is equal to the dimension of the subspace onto which $A$ projects (just look at the normal form of the matrix of the projection), it follows that

a given projection $A$ belongs to $\mathfrak{s l}$ if and only if it is a projection

onto a subspace of even dimension.

If $A \in \mathfrak{s l}(2 n+1)$ is a projection, then the Lie superalgebra obtained from it by "method 2 " of $[7]$ is isomorphic to

$$
\mathfrak{s l}(\operatorname{dim} \operatorname{Ker} A \mid \operatorname{dim} \operatorname{Im} A) \cong \mathfrak{s l}(\operatorname{dim} \operatorname{Im} A \mid \operatorname{dim} \operatorname{Ker} A) .
$$

So we see there are $n+1$ equivalence classes of $\mathbb{Z} / 2$-gradings of $\mathfrak{s l}(2 n+1)$ and $n+1$ of its nonisomorphic superizations (including the trivial purely even one). We can enumerate them either as

$$
\mathfrak{s l}(2 n+1-2 k \mid 2 k) \cong \mathfrak{s l}(2 k \mid 2 n+1-2 k), \quad \text { where } k=0, \ldots, n,
$$

or, more simply, as $\mathfrak{s l}(k \mid 2 n+1-k)$, where $k=0, \ldots, n$. So the answer is the same as for $p \neq 2$.

The $\mathfrak{s l}(2 n)$ series $(n \geq 2)$. The Lie algebra $\mathfrak{d e r} \mathfrak{s l}(2 n)$ can be identified, see [23], with $\mathfrak{p g l}(2 n)$ in the sense that for any $D \in \mathfrak{d e r} \mathfrak{s l}(2 n)$, there is $A_{D} \in \mathfrak{g l}(2 n)$ such that $D$ coincides with the restriction of $\operatorname{ad}_{A_{D}}$ to $\mathfrak{s l}(2 n)$.

These elements $A_{D}$ are defined modulo center; in particular, one can take $A_{D^{2}}$ to be $\left(A_{D}\right)^{2}$. Therefore, $D$ satisfies the condition (1.7a) if and only if $\left(A_{D}\right)^{2}=A_{D}+c I_{2 n}$ for some $c \in \mathbb{K}$. Let $d \in \mathbb{K}$ be a root of the equation $d^{2}=d+c$; set $A_{D}^{\prime}=A_{D}+d I_{2 n}$, then $\left(A_{D}^{\prime}\right)^{2}=A_{D}^{\prime}$. So we see that an operator $U$ on $\mathfrak{s l}(2 n)$ satisfies the conditions (1.7a) if and only if it can be represented as a restriction of $\operatorname{ad}_{A}$ to $\mathfrak{s l}(2 n)$ for some projection $A \in \mathfrak{g l}(2 n)$, and then the Lie superalgebra obtained from $U$ by "method 2" is isomorphic to

$$
\mathfrak{s l}(\operatorname{dim} \operatorname{Ker} A \mid \operatorname{dim} \operatorname{Im} A) \cong \mathfrak{s l}(\operatorname{dim} \operatorname{Im} A \mid \operatorname{dim} \operatorname{Ker} A) .
$$

So there are $n+1$ nonisomorphic superizations of $\mathfrak{s l}(2 n)$ (including the trivial purely even one): $\mathfrak{s l}(k \mid 2 n-k)$, where $k=0, \ldots, n$. Again, the answer is the same as for $p \neq 2$.

The $\mathfrak{p s l}(2 n)$ series, $n>2$. The algebra $\mathfrak{d e r} \mathfrak{p} \mathfrak{s l}(2 n)$ is isomorphic to $\mathfrak{d e r} \mathfrak{s l}(2 n)$ when $n>2$, see [23]. So the arguments from the previous subsection apply. There are $n+1$ non-isomorphic superizations of $\mathfrak{p s l}(2 n)$ (including the purely even one), which can be enumerated as $\mathfrak{p s l}(k \mid 2 n-k)$, where $k=0, \ldots, n$.

Remark 2.3. A $\mathbb{Z} / 2$-grading of $\mathfrak{g l}(2 n+1)$ for $n>2$ can produce a Lie superalgebra which is not isomorphic to any superalgebra of the form $\mathfrak{g l}(k \mid 2 n+1-k)$. More specifically, such a superalgebra would be isomorphic to the direct sum of a Lie superalgebra of the form $\mathfrak{s l}(k \mid 2 n+1-k)$, where $0 \leq k \leq n$, and 0|1-dimensional center (i.e., 1-dimensional odd center). Namely, represent $\mathfrak{g l}(2 n+1)$ as $\mathfrak{s l}(2 n+1) \oplus \mathfrak{c}$; since it is a direct sum, you can introduce gradings on the summands independently. On $\mathfrak{s l}(2 n+1)$, introduce some grading which produces $\mathfrak{s l}(k \mid 2 n+1-k)$, then declare the center odd. The resulting algebra is not isomorphic to $\mathfrak{g l}(k \mid 2 n+1-k)$ for any $k$, since the center of $\mathfrak{g l}(k \mid 2 n+1-k)$ is even. But since this grading does not result in any new simple Lie superalgebra, we do not consider gradings of $\mathfrak{g l}$ here.

\section{The $\mathfrak{o}^{(1)}(2 n+1)$ series}

Clearly, $\mathfrak{o o}_{B_{1} B_{2}}(0 \mid n)=\mathfrak{o}_{B_{2}}(n)$ and $\mathfrak{o o}_{B_{1} B_{2}}(n \mid 0)=\mathfrak{o}_{B_{1}}(n)$. 
Example $3.1\left(\mathfrak{o}^{(1)}(3)\right)$. Observe the occasional isomorphism $\mathfrak{o}^{(1)}(3) \simeq \mathfrak{v e c t} \mathfrak{t}^{(1)}(1 ; \underline{2})$, so this case is considered in more details in Section 6.1. In [6], the deforms of the superization of $\mathfrak{o}^{(1)}(3)$ associated with one of the three $\mathbb{Z} / 2$-gradings of $\mathfrak{o}^{(1)}(3)$ are described; but until now it was unclear if these deforms are true ones, i.e., the deformed algebra is not isomorphic to the initial algebra, as is the case for semitrivial deformations corresponding to certain integrable cocycles from nontrivial cohomology class, for examples, see $[8,24]$. The results of Section 6.1 prove that one of the deforms found in [6] is a true one.

Theorem 3.2. For any $n \geq 1$, all $\mathbb{Z} / 2$-gradings of the Lie algebra $\mathfrak{o}^{(1)}(2 n+1)$ correspond to Lie superalgebras $\mathfrak{o o}_{I I}^{(1)}(2 n+1-2 k \mid 2 k)$, where $0 \leq k \leq n$ (or, which is the same but looks simpler, $\mathfrak{o o}_{I I}^{(1)}(k \mid 2 n+1-k)$, where $\left.0 \leq k \leq n\right)$, and $\mathfrak{o o}_{I \Pi}^{(1)}(2 n+1-2 k \mid 2 k)$, where $1 \leq k \leq n$.

Proof. Since any nondegenerate symmetric bilinear form over a space of dimension $2 n+1$ is equivalent to $I_{2 n+1}$, the algebra of derivations of $\mathfrak{o}^{(1)}(2 n+1)$ is isomorphic to $\mathfrak{o}(2 n+1) / \mathfrak{c}$, the quotient of $\mathfrak{o}(2 n+1)$ modulo center, or the subalgebra of traceless elements of $\mathfrak{o}(2 n+1)$, see [2, Statement 3.8.1a] and Appendix A.

Again, an element $A \in \mathfrak{d e r} \mathfrak{o}^{(1)}(2 n+1)$ describes a $\mathbb{Z} / 2$-grading of $\mathfrak{o}^{(1)}(2 n+1)$ if and only if $A^{2}=A$, i.e., $A$ is a projection.

By definition, an operator $A \in \mathfrak{g l}(V)$ belongs to $\mathfrak{o}_{B}(V)$, where $B$ is a nondegenerate symmetric bilinear form, if and only if

$$
B(A x, y)+B(x, A y)=0 \quad \text { for all } x, y \in V .
$$

In what follows, we assume that $A$ is a projection. It is easy to check that (3.1) is automatically satisfied if $x, y \in \operatorname{Im} A$ or if $x, y \in \operatorname{Ker} A$. If $x \in \operatorname{Im} A$ and $y \in \operatorname{Ker} A$, then (3.1) is equivalent to $B(x, y)=0$. So $A \in \mathfrak{o}_{B}(V)$ if and only if $\operatorname{Im} A$ and $\operatorname{Ker} A$ are orthogonal with respect to $B$. For $A$ to be traceless, $\operatorname{dim} \operatorname{Im} A$ must be even, see condition (2.1).

Let $A$ be a projection belonging to $\mathfrak{d e r} \mathfrak{o}_{B}^{(1)}(2 n+1)$ with $\operatorname{dim} \operatorname{Im} A=2 k>0$. Denote restrictions of $B$ to $\operatorname{Im} A$ and $\operatorname{Ker} A$ by $B_{\operatorname{Im} A}$ and $B_{\operatorname{Ker} A}$, respectively.

Since $B$ is nondegenerate, $\operatorname{Im} A \oplus \operatorname{Ker} A=V$ and $\operatorname{Im} A \perp \operatorname{Ker} A$ with respect to $B$, it follows that these restrictions are nondegenerate.

Since $\operatorname{dim} \operatorname{Ker} A=2 n+1-2 k$ is odd, $B_{\operatorname{Ker} A}$ is equivalent to $I_{2 n+1-2 k}$. Since $\operatorname{dim} \operatorname{Im} A=2 k$ is even, it follows that $B_{\operatorname{Im} A}$ may be equivalent to either $I_{2 k}$, or $\Pi_{2 k}$. In the latter case, the resulting Lie superalgebra is isomorphic to $\mathfrak{o o}_{I \Pi}^{(1)}(2 n+1-2 k \mid 2 k)$; in the former case, it is isomorphic to $\mathfrak{o o}_{I I}^{(1)}(2 k \mid 2 n+1-2 k)$. Note that the collection "ooo ${ }_{I I}^{(1)}(2 k \mid 2 n+1-2 k)$, where $0 \leq k \leq n$ " can be described more simply as "oo $(1)(k \mid 2 n+1-k)$, where $0 \leq k \leq n$ ".

So we get $2 n+1$ nonisomorphic Lie superalgebras from $\mathfrak{o}^{(1)}(2 n+1)$, including the purely even case. To see that all these cases are really realizable with some projection $A$, the following approach can be used. Recall that in an odd-dimensional space, all nondegenerate symmetric forms are equivalent, so for any such form $B$ and any symmetric invertible matrix, there is a basis in which the matrix of $B$ is equal to the given one. In particular, for a given $k$, there are bases in which the Gram matrix of $B$ is either $I_{2 n+1}$ (call it the first basis) or $\operatorname{diag}\left(I_{2 n+1-2 k}, \Pi_{2 k}\right)$ (call it the second basis for this value of $k$ ).

Take the operator $A$ whose matrix in the first basis is $\operatorname{diag}\left(0_{2 n+1-2 k}, I_{2 k}\right)$; clearly, $A^{2}=A$ and $\operatorname{dim} \operatorname{Im} A=2 k$. The space $\operatorname{Im} A$, which is spanned by the last $2 k$ vectors of the basis, is, clearly, orthogonal, relative the form $B$, to $\operatorname{Ker} A$, which is spanned by the first $2 n+1-2 k$ vectors of the basis. So $A \in \mathfrak{d e r} \mathfrak{o}_{B}$, and hence determines a grading of $\mathfrak{o}_{B}^{(1)}$. It is easy to see that the Lie superalgebra obtained from $\mathfrak{o}_{B}^{(1)}$ by "method 2" of [7] is $\mathfrak{o o}_{I I}^{(1)}(2 n+1-2 k \mid 2 k)$.

Analogously, an operator $A$ with the same matrix $\operatorname{diag}\left(0_{2 n+1-2 k}, I_{2 k}\right)$ in the second basis (it does not matter what is the matrix of $A$ in the first basis) determines a grading that corresponds to the Lie superalgebra $\mathfrak{o o}_{I \Pi}^{(1)}(2 n+1-2 k \mid 2 k)$. 
It is possible to show that there are $2 n+1$ equivalence classes of $\mathbb{Z} / 2$-gradings. It could have happened that there were more gradings than nonisomorphic superizations if two inequivalent gradings would have yielded isomorphic Lie superalgebras. This, however, does not happen; we skip the details.

Remark 3.3. Similarly to $\mathfrak{g l}(2 n+1)$, see Remark 2.3 , it is clear that certain $\mathbb{Z} / 2$-gradings of $\mathfrak{o}(2 n+1)$ can produce Lie superalgebras not isomorphic to any superalgebra of the form $\mathfrak{o o}_{I I}(2 n+1-2 k \mid 2 k)$ or $\mathfrak{o o}_{I \Pi}(2 n+1-2 k \mid 2 k)$. For example, certain $\mathbb{Z} / 2$-gradings can yield a direct sum of $\mathfrak{o o}_{I I}^{(1)}(2 n+1-2 k \mid 2 k)$ and $0 \mid 1$-dimensional center. But again, such gradings do not produce any new simple Lie superalgebras, so, being actually interested in new simple Lie superalgebras, we do not consider such gradings of $\mathfrak{o}(2 n+1)$ in full generality in this paper.

\section{The $\mathfrak{o}_{I}^{(1)}(2 n)$ series}

Theorem 4.1. For $n>2$, all $\mathbb{Z} / 2$-gradings of the Lie algebra $\mathfrak{o}_{I}^{(1)}(2 n)$ correspond to Lie superalgebras $\mathfrak{o o}_{I I}^{(1)}(m \mid 2 n-m)$, where $1 \leq m \leq n$, and $\mathfrak{o o}_{I \Pi}^{(1)}(2 n-2 k \mid 2 k)$, where $0 \leq k \leq n-1$.

Proof. If a bilinear form $B$ on vector space $V$ of dimension $2 n$ is equivalent to $I_{2 n}$, then the algebra $\mathfrak{d e r} \mathfrak{o}_{B}^{(1)}(2 n)$ can be identified, see [2, Statement 3.8.1a] and Appendix A, with $\mathfrak{o}_{B}(2 n) / \mathfrak{c}$ in the sense that for any $D \in \mathfrak{d e r} \mathfrak{o}_{B}^{(1)}(2 n)$, there is $A_{D} \in \mathfrak{o}_{B}(2 n)$ such that $D$ coincides with the restriction of $\operatorname{ad}_{A_{D}}$ to $\mathfrak{o}_{B}^{(1)}(2 n)$. These elements $A_{D}$ are defined up to a central element; in particular, one can take $A_{D^{2}}$ to be $\left(A_{D}\right)^{2}$. By arguments similar to the ones we used in the case of $\mathfrak{s l}(2 n)$, one can show that an operator $U$ on $\mathfrak{o}_{B}^{(1)}(2 n)$ satisfies the conditions (1.7a) if and only if it can be represented as a restriction of $\operatorname{ad}_{A}$ to $\mathfrak{o}_{B}^{(1)}(2 n)$ for some projection $A \in \mathfrak{o}_{B}(2 n)$.

By arguments we used in the previous section, a projection $A$ belongs to $\mathfrak{o}_{B}(2 n)$ if and only if $\operatorname{Im} A$ and $\operatorname{Ker} A$ are orthogonal with respect to $B$. We denote restrictions of $B$ to $\operatorname{Im} A$ and Ker $A$ by $B_{\operatorname{Im} A}$ and $B_{\operatorname{Ker} A}$, respectively. These restrictions have to be nondegenerate.

If $\operatorname{dim} \operatorname{Im} A=2 k+1$, where $0 \leq k \leq n-1$, then these restrictions are equivalent to $I_{2 k+1}$ and $I_{2 n-2 k-1}$, respectively, and the resulting Lie superalgebra is isomorphic to

$$
\mathfrak{o}_{I I}^{(1)}(2 k+1 \mid 2 n-2 k-1) \simeq \mathfrak{o}_{I I}^{(1)}(2 n-2 k-1 \mid 2 k+1) .
$$

If $\operatorname{dim} \operatorname{Im} A=2 k$, where $1 \leq k \leq n-1$, then $B_{\operatorname{Im} A}$ can be equivalent to either $I_{2 k}$, or $\Pi_{2 k}$, while $B_{\operatorname{Ker} A}$ can be equivalent to either $I_{2 n-2 k}$, or $\Pi_{2 n-2 k}$. However, it is impossible for $B_{\operatorname{Im} A}$ to be equivalent to $\Pi_{2 k}$ while $B_{\operatorname{Ker} A}$ is equivalent to $\Pi_{2 n-2 k}$ at the same time, because the direct sum of these two forms is equivalent to $\Pi_{2 n}$. All the other combinations are possible, and, depending on them, the resulting Lie superalgebra can be isomorphic to either $\mathfrak{o}_{I I}^{(1)}(2 k \mid 2 n-2 k)$, or $\mathfrak{o}_{I \Pi}^{(1)}(2 k \mid 2 n-2 k)$, or $\mathfrak{o}_{\Pi I}^{(1)}(2 k \mid 2 n-2 k) \simeq \mathfrak{o}_{I \Pi}^{(1)}(2 n-2 k \mid 2 k)$.

If $\operatorname{dim} \operatorname{Im} A=0$ or $2 n$, then the resulting Lie superalgebra is purely even. So, as described above, $\mathfrak{o}_{B}^{(1)}(2 n)$ has $2 n+1$ nonisomorphic superizations.

Remark 4.2. The algebras $\mathfrak{o}_{I}^{(1)}(2)$ and $\mathfrak{o}_{I}^{(1)}(4)$ are not simple, so we do not consider them here.

\section{The simple relatives of $\mathfrak{o}_{\Pi}(2 n)$ series}

The algebras $\mathfrak{o}_{\Pi}(2)$ and $\mathfrak{o}_{\Pi}(4)$ do not have simple relatives, so we do not consider them. We have been unable to classify $\mathbb{Z} / 2$-gradings of the simple relative of $\mathfrak{o}_{\Pi}(8)$ so far. 
Convention. We will denote the simple relative of $\mathfrak{o}_{\Pi}(2 n)$ by $\mathfrak{o}_{\Pi}^{(2)}(2 n) / \mathfrak{c}$ for both even and odd values of $n$, keeping in mind that the center might be trivial. Similarly, we will denote the simple relatives of $\mathfrak{o o}_{\Pi \Pi}(2 k \mid 2 n-2 k)$ and $\mathfrak{p e} \mathfrak{e}^{(2)}(n)$ by $\mathfrak{o o}_{\Pi \Pi}^{(2)}(2 k \mid 2 n-2 k) / \mathfrak{c}$ and $\mathfrak{p e} \mathfrak{e}^{(2)}(n) / \mathfrak{c}$, respectively, for both even and odd values of $n$.

Theorem 5.1. For $n=3$ or $n \geq 5$, all $\mathbb{Z} / 2$-gradings of the simple relative of $\mathfrak{o}_{\Pi}(2 n)$ (that is, of $\mathfrak{o}_{\Pi}^{(2)}(2 n)$, if $n$ is odd, or of $\mathfrak{o}_{\Pi}^{(2)}(2 n) / \mathfrak{c}$, if $n$ is even) correspond to the simple relatives of the corresponding superizations, i.e., $\mathfrak{o o}_{\Pi \Pi}^{(2)}(2 k \mid 2 n-2 k)$, where $0 \leq k \leq\left\lfloor\frac{n}{2}\right\rfloor$, and $\mathfrak{p e}^{(2)}(n)$, if $n$ is odd, or $\mathfrak{o o}_{\Pi \Pi}^{(2)}(2 k \mid 2 n-2 k) / \mathfrak{c}$, where $0 \leq k \leq\left\lfloor\frac{n}{2}\right\rfloor$, and $\mathfrak{p e}^{(2)}(n) / \mathfrak{c}$, if $n$ is even.

Proof. If $n$ is odd, then the center of $\mathfrak{o}_{\Pi}^{(2)}(2 n)$ is trivial, so in this case $\mathfrak{o}_{\Pi}^{(2)}(2 n) / \mathfrak{c} \simeq \mathfrak{o}_{\Pi}^{(2)}(2 n)$.

For a symmetric bilinear form $B$ on space $V$, consider the Lie algebra

$$
\begin{aligned}
\tilde{\mathfrak{o}}_{B}(V):=\{ & \left\{M \in \mathfrak{g l}(V) \mid \text { there is } c_{M} \in \mathbb{K}\right. \text { such that } \\
& \left.B(M x, y)+B(x, M y)=c_{M} B(x, y) \text { for all } x, y \in V\right\} .
\end{aligned}
$$

As is not difficult to see, for $B \sim \Pi_{2 n}$ we have

$$
\tilde{\mathfrak{o}}_{\Pi_{2 n}}(V)=\mathfrak{o}_{\Pi_{2 n}}(V) \ltimes \mathbb{K} d_{n}, \quad \text { where } d_{n}=\operatorname{diag}\left(0_{n}, 1_{n}\right) \text { or } \operatorname{diag}\left(1_{n}, 0_{n}\right) \text {. }
$$

Then, according to $[2,23]$

$$
\mathfrak{d e r}\left(\mathfrak{o}_{\Pi_{2 n}}^{(2)}(V) / \mathfrak{c}\right) \simeq \tilde{\mathfrak{o}}_{\Pi_{2 n}}(V) / \mathfrak{c} .
$$

(To compare with our previous results, observe that if $B \sim I_{2 n}$, then $\tilde{\mathfrak{o}}_{I_{2 n}}(V)=\mathfrak{o}_{I_{2 n}}(V)$.)

As before, a given operator $U \in \mathfrak{d} \mathfrak{e r}\left(\mathfrak{o}_{B}^{(2)}(V) / \mathfrak{c}\right) \simeq \tilde{\mathfrak{o}}_{B}(V) / \mathfrak{c}$ satisfies the condition $U^{2}=U$ if and only if the corresponding element (equivalence class) of $\tilde{\mathfrak{o}}_{B}(V)$ contains a projection $A$. Let us consider the values $c_{A}$, see (5.1), can take.

$c_{A}=\overline{0}$ : In this case, the definition (5.1) is equivalent to the statement that $\operatorname{Im} A$ and $\operatorname{Ker} A$ are orthogonal with respect to $B$. It means that the restrictions of $B$ to $\operatorname{Im} A$ and $\operatorname{Ker} A$ have to be nondegenerate. Since $B$ is anti-symmetric (i.e., $B(x, x)=0$ for any $x \in V$ ), these restrictions are anti-symmetric as well, which means that dimensions of $\operatorname{Im} A$ and $\operatorname{Ker} A$ are even. The resulting Lie superalgebra in this case is isomorphic to

$$
\mathfrak{o o}_{\Pi \Pi}^{(2)}(\operatorname{dim} \operatorname{Im} A \mid \operatorname{dim} \operatorname{Ker} A) / \mathfrak{c} \simeq \mathfrak{o o}_{\Pi \Pi}^{(2)}(\operatorname{dim} \operatorname{Ker} A \mid \operatorname{dim} \operatorname{Im} A) / \mathfrak{c} .
$$

$c_{A}=\overline{1}$ : In this case, the condition (5.1) is equivalent to the statement that both $\operatorname{Im} A$ and Ker $A$ are isotropic with respect to $B$. Since $\operatorname{Im} A \oplus \operatorname{Ker} A=V$, this means that

$$
\operatorname{dim} \operatorname{Im} A=\operatorname{dim} \operatorname{Ker} A=n
$$

and there is an invertible linear map $f: \operatorname{Ker} A \rightarrow(\operatorname{Im} A)^{*}$ such that

$$
B(x, y)=(f(y))(x) \quad \text { for all } x \in \operatorname{Im} A, y \in \operatorname{Ker} A \text {. }
$$

The resulting Lie superalgebra in this case is isomorphic to $\mathfrak{p e} \mathfrak{e}^{(2)}(n) / \mathfrak{c}$.

$c_{A} \neq \overline{0}, \overline{1}$ : This is impossible, because in this case, (5.1) would mean that $\operatorname{Im} A$ and $\operatorname{Ker} A$ are both isotropic and orthogonal to each other with respect to $B$, i.e., $B(x, y)=0$ for all $x, y \in V$.

So we get $\left\lfloor\frac{n}{2}\right\rfloor+2$ nonisomorphic superizations of $\mathfrak{o}_{\Pi}^{(2)}(2 n) / \mathfrak{c}$ (including the purely even one), which are $\mathfrak{o o}_{\Pi \Pi}^{(2)}(2 k \mid 2 n-2 k) / \mathfrak{c}$, where $0 \leq k \leq\left\lfloor\frac{n}{2}\right\rfloor$, and $\mathfrak{p} \mathfrak{e}^{(2)}(n) / \mathfrak{c}$. 
Remark 5.2. The algebra $\mathfrak{o}_{\Pi}^{(2)}(6)$ is isomorphic to $\mathfrak{p} \mathfrak{r l}(4), \mathfrak{h}_{\Pi}^{(1)}(4 ; \mathbb{1})$ and $\mathfrak{s v e c t} \mathfrak{c t}^{(1)}(3 ; \mathbb{1})$, see [10]. The fact that it has exactly three superizations implies two facts: 1) the following superizations of these algebras are isomorphic, and 2) these algebras have no other non-trivial superizations non-isomorphic to these ones:

$$
\begin{aligned}
& \mathfrak{o o}_{\Pi \Pi}^{(2)}(2 \mid 4) \simeq \mathfrak{s v e c t}^{(1)}(0 \mid 3) \simeq \mathfrak{p s l}(3 \mid 1) \simeq \mathfrak{s v e c t}^{(1)}(2 ; \mathbb{1} \mid 1), \quad \text { here } \quad \text { sdim }=8 \mid 6, \\
& \mathfrak{p e}^{(2)}(3) \simeq \mathfrak{h}_{\Pi}^{(1)}(0 \mid 4) \simeq \mathfrak{p s l}(2 \mid 2) \simeq \mathfrak{h}_{\Pi \Pi}^{(1)}(2 ; \mathbb{1} \mid 2) \simeq \mathfrak{s v e c t}^{(1)}(1 ; \mathbb{1} \mid 2), \quad \text { here } \quad \text { sdim }=6 \mid 8 .
\end{aligned}
$$

\section{The $\mathfrak{v e c t} \mathfrak{t}^{(1)}(1 ; \underline{n})$ series}

We describe the $\mathbb{Z} / 2$-gradings of $\mathfrak{v e c t}^{(1)}(1 ; \underline{n-1})$ in Theorem 6.2. First, we need some definitions.

Let $\mathfrak{v}_{n}$ be the minimal Lie subalgebra of the restricted closure of $\mathfrak{v e c t}^{(1)}(1 ; \underline{n-1})$ that contains $\mathfrak{v e c t}^{(1)}(1 ; \underline{n-1})$ and all the elements $\mathcal{X}^{[2]}$, where $\mathcal{X} \in \mathfrak{v e c t}^{(1)}(1 ; \underline{n-1})$. As a vector space, $\mathfrak{v}_{n}$ can be written as a direct sum

$$
\mathfrak{v}_{n}=\mathfrak{v e c t}(1 ; \underline{n-1}) \oplus \mathbb{K} \partial^{2} .
$$

Thus, $\operatorname{dim} \mathfrak{v}_{n}=2^{n-1}+1$. Observe that $\mathfrak{v}_{n}=\left(\mathfrak{v e c t}^{(1)}(1 ; \underline{n-1})\right)^{\langle 1\rangle}$, see equation (1.5), associated with the $\mathbb{Z} / 2$-grading induced by the standard $\mathbb{Z}$-grading of $\mathfrak{v e c t}^{(1)}(1 ; \underline{n-1})$.

For $n>2$, consider the Lie superalgebra $\mathfrak{q}(\mathfrak{v e c t}(1 ; \underline{n-1}))$. As a vector space, it can be written as a direct sum $\mathfrak{v}_{n} \oplus \Pi(\mathfrak{v e c t}(1 ; \underline{n-1}))$, where $\Pi$ is the change of parity functor. Consider the Lie algebra $\mathfrak{v e c t}(1 ; \underline{n})$ in an indeterminate $z$ in order to distinguish it from the indeterminate $x$ in equation (6.3). Let

$$
X_{-2}=\partial^{2}, X_{-1}=\partial, X_{0}=z \partial, \ldots, X_{2^{n-1}-2}=z^{2^{n-1}-1} \partial,
$$

be the basis in the even part $\mathfrak{v}_{n}$ of $\mathfrak{q}(\mathfrak{v e c t}(1 ; \underline{n-1}))$ and

$$
Y_{-1}=\Pi \partial, Y_{0}=\Pi(z \partial), \ldots, Y_{2^{n-1}-2}=\Pi\left(z^{\left(2^{n-1}-1\right)} \partial\right),
$$

be the basis in the odd part of $\mathfrak{q}(\mathfrak{v e c t}(1 ; \underline{n-1}))$. The Lie superalgebra structure in $\mathfrak{q}(\mathfrak{v e c t}(1 ; \underline{n-1}))$ is given by following formulas

$$
\begin{aligned}
& {\left[X_{-2}, X_{k}\right]=\left[\partial^{2}, z^{(k-1)} \partial\right]=X_{k-2} \quad \text { for } k=1, \ldots, 2^{n-1}-2,} \\
& {\left[X_{-2}, Y_{k}\right]=\Pi\left[\partial^{2}, z^{(k-1)} \partial\right]=Y_{k-2} \quad \text { for } k=1, \ldots, 2^{n-1}-2,} \\
& {\left[X_{-2}, X_{-1}\right]=\left[X_{-2}, X_{0}\right]=\left[X_{-2}, Y_{-1}\right]=\left[X_{-2}, Y_{0}\right]=\left[X_{-1}, Y_{-1}\right]=0,} \\
& {\left[X_{k}, X_{m}\right]=\left[z^{(k-1)} \partial, z^{(m-1)} \partial\right]=\left(\begin{array}{c}
k+m+2 \\
k+1
\end{array}\right) X_{k+m}} \\
& \quad \text { for } k, m=-1, \ldots, 2^{n-1}-2, \text { except for } m=k=-1, \\
& {\left[X_{k}, Y_{m}\right]=\Pi\left[z^{(k-1)} \partial, z^{(s-1)} \partial\right]=\left(\begin{array}{c}
k+m+2 \\
k+1
\end{array}\right) Y_{k+m}} \\
& \quad \text { for } k, m=-1, \ldots, 2^{n-1}-2, \text { except for } m=k=-1, \\
& {\left[Y_{k}, Y_{m}\right]=\left[z^{(k-1)} \partial, z^{(m-1)} \partial\right]=\left(\begin{array}{c}
k+m+2 \\
k+1
\end{array}\right) X_{k+m}} \\
& \quad \text { for } k, m=-1, \ldots, 2^{n-1}-2, \text { except for } m=k=-1, \\
& \left(Y_{-1}\right)^{2}=X_{-2}, \quad \text { for } k=0, \ldots, 2^{n-1}-2 . \\
& \left(Y_{k}\right)^{2}=\left(z^{(k+1)} \partial\right)^{[2]}=\left(\begin{array}{c}
2 k+1 \\
k
\end{array}\right) X_{2 k}
\end{aligned}
$$


On the superspace of $\mathfrak{q}(\mathfrak{v} \mathfrak{e c t}(1 ; \underline{n-1}))$, define another Lie superalgebra structure, call it $\widetilde{\mathfrak{q v}}_{n-1}$, by the following formulas:

$$
\begin{aligned}
& {\left[X_{-2}, X_{-1}\right]:=0, \quad\left[X_{-2}, X_{0}\right]:=0,} \\
& {\left[X_{-2}, X_{m}\right]:=X_{m-2} \quad \text { for } m=0, \ldots, 2^{n-1}-2,} \\
& {\left[X_{k}, X_{m}\right]:=\left(\begin{array}{c}
k+m+2 \\
k+1
\end{array}\right) X_{k+m} \quad \text { for } m, k=-1, \ldots, 2^{n-1}-2, \text { except for } m=k=-1,} \\
& {\left[Y_{k}, Y_{m}\right]:=\left(\begin{array}{c}
k+m+2 \\
k+1
\end{array}\right) X_{k+m} \quad \text { for } m, k=-1, \ldots, 2^{n-1}-2, \text { except for } m=k=-1,} \\
& {\left[X_{-1}, Y_{-1}\right]:=Y_{-1}, \quad\left[X_{-2}, Y_{-1}\right]=Y_{-1}, \quad\left[X_{-2}, Y_{0}\right]=Y_{0},} \\
& {\left[X_{-2}, Y_{m}\right]=Y_{m}+Y_{m-2}, \quad m=1, \ldots, 2^{n-1}-2} \\
& {\left[X_{k}, Y_{m}\right]:=\left(\begin{array}{c}
k+m+2 \\
k+1
\end{array}\right) Y_{k+m}+\left(\begin{array}{c}
k+m+2 \\
k+1
\end{array}\right) Y_{k+m+1}} \\
& \quad \text { for } k, m=-1, \ldots, 2^{n-1}-2, \text { except for } m=k=-1, \\
& \left(Y_{-1}\right)^{2}:=X_{-2}+X_{0}, \quad\left(Y_{k}\right)^{2}:=\left(\begin{array}{c}
2 k+1 \\
k
\end{array}\right) X_{2 k} \quad \text { for } k=0, \ldots, 2^{n-1}-2 .
\end{aligned}
$$

I. Shchepochkina observed that the Lie superalgebra $\widetilde{\mathfrak{q v}}_{n-1}$ is a filtered deform of the Lie superalgebra $\mathfrak{q}(\mathfrak{v e c t}(1 ; \underline{n-1}))$.

Lemma 6.1. Lie superalgebras $\widetilde{\mathfrak{q v}}_{n-1}$ and $\mathfrak{q}(\mathfrak{v e c t}(1 ; \underline{n-1}))$ are not isomorphic.

Proof. Let us prove that $\widetilde{\mathfrak{q v}}_{n-1}$ is not isomorphic to any Lie superalgebra of the form $\mathfrak{q}(\mathfrak{g})$, where $\mathfrak{g}$ is a Lie algebra. Any superalgebra of the form $\mathfrak{q}(\mathfrak{g})$ possesses the following property: if $x \in \mathfrak{q}(\mathfrak{g})_{\overline{0}}$ acts nilpotently on $\mathfrak{q}(\mathfrak{g})_{\overline{0}}$, then it acts nilpotently on $\mathfrak{q}(\mathfrak{g})_{\overline{1}}$ as well. Indeed, if $\left.\left(\operatorname{ad}_{x}\right)^{k}\right|_{\mathfrak{q}(\mathfrak{g})_{\overline{0}}}=0$ for some positive integer $k$, then $\left(\operatorname{ad}_{x}\right)^{k} \Pi y=\Pi\left(\left(\operatorname{ad}_{x}\right)^{k} y\right)=0$ for any $y \in \mathfrak{g}$.

On the other hand, from the relations above one can see that $X_{-1}$ acts nilpotently on $\left(\widetilde{\mathfrak{q v}}_{n-1}\right) \overline{0}$ (more specifically, $\left.\left.\left(\operatorname{ad}_{X_{-1}}\right)^{2^{n-1}+1}\right|_{\left(\widetilde{\mathfrak{q}}_{n-1}\right)_{\overline{0}}}=0\right)$ ), but $\left[X_{-1}, Y_{-1}\right]=Y_{-1}$, so the action of $X_{-1}$ on $\left(\widetilde{\mathfrak{q v}}_{n-1}\right)_{\overline{1}}$ is not nilpotent.

Consider the Lie algebra $\mathfrak{v e c t}(1 ; \underline{n})$ in an indeterminate $x$. For a basis of $\mathfrak{v e c t}(1 ; \underline{n})$ we take $\boldsymbol{e}_{i}:=x^{(i+1)} \partial$ for $i=-1,0, \ldots, 2^{n}-2$.

Theorem 6.2. All $\mathbb{Z} / 2$-gradings of the Lie algebra $\mathfrak{v e c t}^{(1)}(1 ; \underline{n})$ correspond to the one of the following Lie superalgebras:

1) purely even $\mathfrak{v e c t}^{(1)}(1 ; \underline{n} \mid 0)$;

2) $\mathfrak{k}(1 ; \underline{n-1} \mid 1)$, described in [3], and its $(n-2)$-parametric family of filtered deforms described below in the proof;

3) the Lie superalgebra $\widetilde{\mathfrak{q v}}_{n-1}$ for $n>2$.

Proof. Let $u \in \mathcal{O}(1 ; \underline{n})$ be a linear combination of only even powers of the indeterminate $x$, and let $a$ be its constant term ${ }^{2}$. In other words, let $u, a \in \mathcal{O}(1 ; \underline{n})$ be such that

$$
x \cdot(\partial u)=0, \quad \partial a=0, \quad u^{2}=a^{2} .
$$

\footnotetext{
${ }^{2}$ The notation $u(0)$ we use for brevity in what follows is meaningless, strictly speaking, because the divided power polynomials can not be evaluated at any $x \in \mathbb{K}$ for $p>0$; by writing like this we mean $a \in \mathbb{K} \cdot 1$ which is the value of $u$ modulo the maximal ideal of $\mathcal{O}(1 ; \underline{n})$; this is a standard abuse of notation.
} 
Consider any derivation $D_{u} \in \mathfrak{d e r} \mathcal{O}(1 ; \underline{n})$ of the form

$$
D_{u}=\left(u+x+x u \sum_{1 \leq i \leq n-1} a^{2^{i}-2}\left(\partial^{2^{i}} u\right)\right) \partial+\sum_{1 \leq i \leq n-1} a^{2^{i}} \partial^{2^{i}} .
$$

Properties (6.2) imply (after rather lengthy calculations we omit) that $\left(D_{u}\right)^{2}=D_{u}$, so $D_{u}$ describes a $\mathbb{Z} / 2$-grading of $\mathcal{O}(1 ; \underline{n})$.

Consider the linear map $\operatorname{ad}_{D_{u}}$ on the Lie algebra $\mathfrak{d e r} \mathcal{O}(1 ; \underline{n})$ given by $D \mapsto\left[D_{u}, D\right]$. Due to the Jacobi identity, we see that $\operatorname{ad}_{D_{u}} \in \mathfrak{d e r}(\mathfrak{d e r} \mathcal{O}(1 ; \underline{n}))$, where the outer $\mathfrak{d e r}$ is for derivations of the Lie algebra $\mathfrak{d e r} \mathcal{O}(1 ; \underline{n})$. Since the Lie algebra $\mathfrak{d e r} \mathcal{O}(1 ; \underline{n})$ has a 2 -structure given by $D^{[2]}=D^{2}$, we have $\left(\operatorname{ad}_{D_{u}}\right)^{2}=\operatorname{ad}_{D_{u}}$. Thus, $\operatorname{ad}_{D_{u}}$ describes a $\mathbb{Z} / 2$-grading of $\mathfrak{d e r} \mathcal{O}(1 ; \underline{n})$.

The linear map $\operatorname{ad}_{D_{u}}$ sends elements of $\mathfrak{v e c t}(1 ; \underline{n})$ to $\mathfrak{v e c t}^{(1)}(1 ; \underline{n})$ because $D_{u}$ is a linear combination of a vector field and elements of the form $\partial^{2^{i}}$, and

$$
\left[\partial^{2^{i}}, f \partial\right]=\left(\partial^{2^{i}} f\right) \partial=\left[\partial,\left(\partial^{2^{i}-1} f\right) \partial\right] \in \mathfrak{v e c t}^{(1)}(1 ; \underline{n}) \quad \text { for any } f \in \mathcal{O}(1 ; \underline{n}) .
$$

This means that the restriction $\left.\left(\operatorname{ad}_{D_{u}}\right)\right|_{\mathfrak{v e c t}(1 ; \underline{n})}$ describes a $\mathbb{Z} / 2$-grading of $\mathfrak{v e c t}(1 ; \underline{n})$ and the restriction $\left.\left(\operatorname{ad}_{D_{u}}\right)\right|_{\mathfrak{v e c t}(1)(1 ; \underline{n})}$ describes a $\mathbb{Z} / 2$-grading of $\mathfrak{v e c t}^{(1)}(1 ; \underline{n})$. In what follows, we will denote the latter restriction by $D_{u}$ as well. Different polynomials $u$ correspond to different gradings, as $\left[D_{u}, x \partial\right]=u \partial$. We need, however, not individual gradings, but their equivalence classes. We were unable to solve this problem completely so far.

In notation of [29], any torus of the restricted closure of $\mathfrak{v e c t}(1 ; \underline{n})$ lying in the maximal subalgebra of elements of non-negative degree (assuming $\operatorname{deg} x=1$ ) is called an inner one, the other tori are called outer ones.

If $u(0)=a \neq 0$, the derivation $D_{u}$ is an outer toroidal derivation of $\mathcal{O}(1 ; \underline{n})$ (i.e., it spans an outer torus of 2 -closure of $\mathfrak{v e c t}(1 ; \underline{n}))$. The automorphism group of $\mathfrak{v e c t}^{(1)}(1 ; \underline{n})$ acts transitively on the set of outer toroidal derivations, see [29]. ${ }^{3}$ Hence, any derivation $D_{u}$ with $a \neq 0$ is conjugate by an automorphism of $\mathfrak{v e c t} \mathfrak{t}^{(1)}(1 ; \underline{n})$ to the derivation

$$
D_{1}=(1+x) \partial+\sum_{1 \leq i \leq n-1} \partial^{2^{i}}
$$

Recall that $(u+v)^{(k)}=\sum_{0 \leq i \leq k} u^{(k-i)} v^{(i)}$ and for $k=-2, \ldots, 2^{n-1}-2$, define $e_{k} \in\left(\mathfrak{v e c t}{ }^{(1)}(1 ; \underline{n}) ; D_{1}\right)^{\langle 1\rangle}$ by setting

$$
\begin{aligned}
& e_{-2}=\partial^{2}+(1+x) \partial, \\
& e_{k}=\partial\left(\left(x+x^{(2)}\right)^{(k+2)}\right) \partial=(1+x)\left(x+x^{(2)}\right)^{(k+1)} \partial \quad \text { for } k>-2,
\end{aligned}
$$

and for $k=-1, \ldots, 2^{n-1}-2$, define $o_{k}$ by formulas

$$
o_{k}=\left(x+x^{(2)}\right)^{(k+1)} \partial, \quad \text { where } k=-1, \ldots, 2^{n-1}-2 .
$$

\footnotetext{
${ }^{3}$ In [29], Tyurin proved that, for any two outer toroidal derivations $O_{1}$ and $O_{2}$ of $\mathfrak{v e c t}(1 ; \underline{n})$, there exists an $A \in \operatorname{Aut}(\mathfrak{v e c t}(1 ; \underline{n}))$ such that $O_{2}=A^{-1} O_{1} A$. Instead, we need "for any two outer toroidal derivations $O_{1}$ and $O_{2}$ of $\mathfrak{v e c t}^{(1)}(1 ; \underline{n})$, there exists an $A \in \operatorname{Aut}\left(\mathfrak{v e c t}^{(1)}(1 ; \underline{n})\right)$ such that $O_{2}=A^{-1} O_{1} A^{\prime \prime}$. This is so because "our" $D_{u}=\left.\left(D_{u}^{T}\right)\right|_{\mathfrak{v e c t}(1)} \in \mathfrak{d e r}_{\mathfrak{v e c t}}{ }^{(1)}(1 ; \underline{n})$ is the restriction of the corresponding "Tyurin's" $D_{u}^{T} \in \mathfrak{d e r} \mathfrak{v e c t}(1 ; \underline{n})$ to $\mathfrak{d e r} \mathfrak{v e c t} \mathfrak{t}^{(1)}(1 ; \underline{n})$.

If $D_{u}$ and $D_{v}$ are outer toroidal derivations of $\mathfrak{v e c t}{ }^{(1)}(1 ; \underline{n})$, then $D_{u}^{T}$ and $D_{v}^{T}$ are outer toroidal derivations of $\mathfrak{v e c t}(1 ; \underline{n})$, and therefore there exists an $A \in \operatorname{Aut}(\mathfrak{v e c t}(1 ; \underline{n}))$ such that $D_{v}^{T}=A^{-1} D_{u}^{T} A$. Any automorphism of $\mathfrak{v e c t}(1 ; \underline{n})$ preserves $\mathfrak{v e c t}^{(1)}(1 ; \underline{n})$, and hence $D_{v}=(\bar{A})^{-1} D_{u} \bar{A}$, where $\bar{A}:=\left.A\right|_{\mathfrak{v e c t}(1)}(1 ; \underline{n})$ is an automorphism of $\mathfrak{v e c t}^{(1)}(1 ; \underline{n})$.
} 
Direct computations show that $D_{1}\left(e_{k}\right)=0$ and $D_{1}\left(o_{m}\right)=o_{m}$. Let $e_{-2}, \ldots, e_{2^{n-1}-2}$ and $\Pi o_{-1}, \ldots, \Pi o_{2^{n}-2}$ form a basis in the Lie superalgebra corresponding to the $\mathbb{Z} / 2$-grading defined by $D_{1}$. The formulas

$$
\begin{aligned}
& e_{k} \mapsto X_{k}, \quad \text { where } k=-2, \ldots, 2^{n-1}-2, \\
& o_{m} \mapsto Y_{m}, \quad \text { where } m=-1, \ldots, 2^{n-1}-2,
\end{aligned}
$$

establish an isomorphism between this Lie superalgebra, spanned by the $e_{i}$ and $o_{j}$, and $\widetilde{\mathfrak{q v}}_{n-1}$.

If $u(0)=a=0$, the derivation $D_{u}$ spans an inner torus. Hence, see [29], the derivation $D_{u}$ is conjugate to the derivation $D_{f}$, where $f=\sum_{1 \leq i \leq n-1} c_{i} x^{\left(2^{i}\right)}$ and the summands $c_{i} x^{\left(2^{i}\right)}$ are the corresponding terms of $u$. This gives us an $(n-1)$-parametric family of $\mathbb{Z} / 2$-gradings. Let the automorphism $\sigma_{\varepsilon}$ of $\mathcal{O}(1 ; \underline{n})$ be given on its generators by the formulas

$$
\sigma_{\varepsilon}(x)=\varepsilon x, \quad \sigma_{\varepsilon}\left(x^{(2)}\right)=\varepsilon^{2} x^{(2)}, \quad \ldots, \quad \sigma_{\varepsilon}\left(x^{\left(2^{n-1}\right)}\right)=\varepsilon^{2^{n-1}} x^{\left(2^{n-1}\right)},
$$

where $\varepsilon \in \mathbb{K}^{\times}$. Then

$$
\sigma_{\varepsilon}\left(\left(\sum_{1 \leq i \leq n-1} c_{i} x^{\left(2^{i}\right)}\right) \partial\right)=\left(\sum_{1 \leq i \leq n-1} c_{i} \varepsilon^{2^{i}-1} x^{\left(2^{i}\right)}\right) \partial .
$$

Hence, the conjugacy class of the derivation $D_{u}$ is defined by a tuple of parameters $\left(c_{1}, c_{2}, \ldots\right.$, $c_{n-1}$ ) up to an equivalence of the form

$$
\left(c_{1}, c_{2}, \ldots, c_{n-1}\right) \sim\left(\varepsilon c_{1}, \varepsilon^{3} c_{2}, \ldots, \varepsilon^{2^{n-1}-1} c_{n-1}\right) .
$$

Therefore, the number of parameters of $\mathbb{Z} / 2$-gradings reduces to $n-2$. In particular, for $n=2$, we do not, indeed, have parametric families of $\mathbb{Z} / 2$-gradings, see Section 6.1.

Observe that $x \cdot \partial u=0$, so

$$
0=\partial(x \partial u)=\partial u+x \partial^{2} u, \quad \text { which implies } \quad x \partial^{2} u=\partial u \text {. }
$$

Then

$$
D_{u}=\left(u+x+x u \partial^{2} u\right) \partial=(u+x+u \partial u) \partial .
$$

Desuperization of any superization of $\mathfrak{v e c t}^{(1)}(1 ; \underline{n})$ can be considered as a Lie subalgebra in $\mathfrak{v}_{n+1}:=\left(\mathfrak{v e c t}^{(1)}(1 ; \underline{n})\right)^{\langle 1\rangle}$, see $(6.1)$. The operator $\operatorname{ad}_{D_{u}}$ preserves $\mathfrak{v}_{n+1}$, so the grading it defines on $\mathfrak{v e c t}^{(1)}(1 ; \underline{n})$ can be extended to $\mathfrak{v}_{n+1}$ with the same definition: $D \mapsto\left[D_{u}, D\right]$. This extended operator describes a $\mathbb{Z} / 2$-grading of $\mathfrak{v}_{n+1}$. The elements of $\mathfrak{v}_{n+1}$ are said to be $u$-even or $u$-odd if they are even or odd, respectively, in this grading.

In particular, for the grading on $\mathfrak{v}_{n+1}$ given by the function $u=0$, we have $D_{0}=x \partial$, and the resulting superization is isomorphic to $\mathfrak{k}(1 ; \underline{n-1} \mid 1)$, see [7, Section 7].

Consider the linear maps $T_{u}, A_{u}: \mathfrak{v}_{n+1} \longrightarrow \mathfrak{v}_{n+1}$ defined as follows (their action on other elements being extended by linearity):

$$
\begin{aligned}
& T_{u}(f \partial)=(f+u(1+\partial u) \partial f)\left(1+\partial u+u \partial^{2} u\right) \partial \quad \text { for any } f \in \mathcal{O}(1 ; \underline{n}), \\
& T_{u} \partial^{2}=\left(T_{u} \partial\right)^{2}=\partial^{2}+\left(\partial^{2} u+u \partial^{3} u+u\left(\partial^{2} u\right)^{2}+u \partial u\left(\partial^{2} u\right)^{2}\right) \partial, \\
& A_{u}(f \partial)=(f+u \partial u \partial f) \partial \quad \text { for any } f \in \mathcal{O}(1 ; \underline{n}), \quad A_{u} \partial^{2}=\partial^{2} .
\end{aligned}
$$

For any $X \in \mathfrak{v}_{n+1}$, one can check that $T_{u} X$ is $u$-even (resp. $u$-odd) if and only if $X$ is 0 -even (resp. 0-odd). We omit the calculations that show it, but the idea is as follows: $\partial$ is 0-odd, 
while $\left(1+\partial u+u \partial^{2} u\right) \partial$ is $u$-odd. Besides, if we extend the concept of $u$-evenness $/ u$-oddness to $\mathcal{O}(1 ; \underline{n})$, then $f+u(1+\partial u) \partial f$ is $u$-even (resp. $u$-odd) for any $f \in \mathcal{O}(1 ; \underline{n})$ if and only if $f$ is 0-even (resp. 0-odd).

Also, the following is true for any $X, Y \in \mathfrak{v e c t}(1 ; \underline{n})$ :

$$
\begin{aligned}
& {\left[T_{u} X, T_{u} Y\right]=T_{u} A_{u}[X, Y], \quad\left(T_{u} X\right)^{2}=T_{u} A_{u} X^{2} \quad \text { for any 0-odd } X,} \\
& {\left[T_{u} \partial^{2}, T_{u} X\right]=T_{u}\left(\left(1+u \partial^{2} u\right) \cdot\left[\partial^{2}, X\right]\right) .}
\end{aligned}
$$

Note also that the operator $T_{u}$ is invertible, so its image is the whole $\mathfrak{v}_{n+1}$. The invertibility of $T_{u}$ follows from the fact that the matrix of $T_{u}$ is upper triangular with 1 's on the main diagonal in the basis $\partial^{2}, \partial, x \partial, \ldots, x^{\left(2^{n}-1\right)} \partial$.

This means that the superization given by any function $u$ such that $u(0)=0$ can be considered as a deform of the superization given by $u=0$ with the deformation parametrized by the polynomial $u$ or, which is the same, the coefficients of $u$ as follows:

$$
\begin{aligned}
& {[X, Y]_{u}= \begin{cases}A_{u}[X, Y] & \text { if } X, Y \in \mathfrak{v e c t}(1 ; \underline{n}), \\
\left(1+u \partial^{2} u\right) \cdot[X, Y] & \text { if } X=\partial^{2} \text { and } Y \in \mathfrak{v e c t}(1 ; \underline{n}), \\
\text { defined by linearity and anti-symmetry } & \text { in other cases, }\end{cases} } \\
& \left(X^{2}\right)_{u}=A_{u} X^{2} \quad \text { for 0-odd } X .
\end{aligned}
$$

This deformation is filtered relative the decreasing filtration in which $\mathcal{L}_{-2}=\mathfrak{s}\left(\mathfrak{v}_{n+1}\right)$, see (1.6), while $\mathcal{L}_{k-1}$ for $k \geq-1$ consists of vector fields of the form $f \partial$, where $f \in \mathcal{O}(1 ; \underline{n})$ does not contain term of degree $<k$. Such superizations are listed in heading 2) of Theorem 6.2.

Finally, observe that these formulas do not capture the trivial $\mathbb{Z} / 2$-grading given by the derivation $U=0$; the even part $\mathfrak{g}_{\overline{0}}$ of this grading is the whole Lie algebra $\mathfrak{v e c t}^{(1)}(1 ; \underline{n})$. Since any torus in $\mathfrak{d e r}\left(\mathfrak{v e c t} \mathfrak{t}^{(1)}(1 ; \underline{n})\right)$ is of the form $D_{u}$, see [21, 29] and footnote 3 on p. 13, we completely described all $\mathbb{Z} / 2$-gradings of $\mathfrak{v e c t}^{(1)}(1 ; \underline{n})$ and the corresponding superizations.

Remark 6.3. Observe an unpredicted fact: according to equations (6.6), if $X, Y \in \mathfrak{v e c t}(1 ; \underline{n})$, then $[X, Y]_{u}$ can be expressed in terms of $[X, Y]$ and $u$.

In particular, concerning the 0 -even part of $\mathfrak{v e c t}(1 ; \underline{n})$, this implies the following fact:

Corollary 6.4. If $u(0)=0$, then the even part of the superization corresponding to $D_{u}$ is a solvable Lie algebra. ${ }^{4}$

For $n=2$, any outer torus is conjugate to an inner one, see (6.10). This is not so for $n>2$.

Lemma 6.5. Let $n>2$, consider the superization $\widetilde{\mathfrak{q v}}_{n-1}=\mathfrak{g}_{\overline{0}} \oplus \mathfrak{g}_{\overline{1}}$ corresponding to the outer torus $D_{1}$. Its even part $\mathfrak{g}_{\overline{0}} \simeq \mathfrak{v}_{n}$ is spanned by the elements (6.3), and $\mathfrak{g}_{\overline{1}}$ is spanned by the elements (6.4). The odd part $\mathfrak{g}_{\overline{1}}$ is a reducible $\mathfrak{g}_{\overline{0}}$-module with no lowest weight vector and with the two highest weight vectors $\Pi_{2^{n-1}-3}$ and $\Pi o_{2^{n-1}-2}$ with respect to the standard $\mathbb{Z}$-grading of $\mathfrak{g}_{\overline{0}}$, namely $\left(\mathfrak{g}_{0}\right)_{k}=\mathbb{K} e_{k}$ for $k=-2, \ldots, 2^{n-1}-2$.

Proof. Using (6.3) and (6.4), for positive generators $e_{2^{k}-1}$, where $k=1, \ldots, n-2$, of $\mathfrak{g}_{\overline{0}}$ we have

$$
\begin{aligned}
{\left[e_{2^{k}-1}, \Pi o_{2^{n-1}-2}\right] } & =\Pi\left[(\partial w) w^{\left(2^{k}\right)} \partial, w^{\left(2^{n-1}-1\right)} \partial\right] \\
& =\Pi\left(w^{\left(2^{k}\right)} w^{\left(2^{n-1}-2\right)}+w^{\left(2^{k}\right)} w^{\left(2^{n-1}-1\right)}+w^{\left(2^{k}-1\right)} w^{\left(2^{n-1}-1\right)}\right) \partial,
\end{aligned}
$$

\footnotetext{
${ }^{4}$ For more examples of simple Lie superalgebras whose even parts are solvable, see [6]. This is a phenomenon indigenous to $p=2$.
} 
where $w=\left(x+x^{(2)}\right)$. Note that

$$
\begin{aligned}
& 2^{n-1}-1=1 \cdot 2^{n-2}+1 \cdot 2^{n-3}+\cdots+1 \cdot 2+1, \\
& 2^{n-1}-2=1 \cdot 2^{n-2}+1 \cdot 2^{n-3}+\cdots+1 \cdot 2+0 .
\end{aligned}
$$

Then by Lucas theorem for $k<n-1$, we have the following equalities, where the marked factor occupies $(k+1)$ st position from the right in the first two lines, while it stands in the $k$ th position from the right in the third line, where the mark is under the place at which the $1 \mathrm{~s}$ in the bottom of the binomial coefficients start to appear

$$
\begin{aligned}
& \left(\begin{array}{c}
2^{n-1}-2+2^{k} \\
2^{k}
\end{array}\right) \bmod 2=\left(\begin{array}{l}
1 \\
0
\end{array}\right)\left(\begin{array}{l}
0 \\
0
\end{array}\right) \cdots\left(\begin{array}{l}
0 \\
0
\end{array}\right) \underbrace{\left(\begin{array}{l}
0 \\
1
\end{array}\right)}\left(\begin{array}{l}
1 \\
0
\end{array}\right) \cdots\left(\begin{array}{l}
1 \\
0
\end{array}\right)\left(\begin{array}{l}
0 \\
0
\end{array}\right)=0, \\
& \left(\begin{array}{c}
2^{n-1}-1+2^{k} \\
2^{k}
\end{array}\right) \bmod 2=\left(\begin{array}{l}
1 \\
0
\end{array}\right)\left(\begin{array}{l}
0 \\
0
\end{array}\right) \cdots\left(\begin{array}{l}
0 \\
0
\end{array}\right) \underbrace{\left(\begin{array}{l}
0 \\
1
\end{array}\right)}\left(\begin{array}{l}
1 \\
0
\end{array}\right) \cdots\left(\begin{array}{l}
1 \\
0
\end{array}\right)\left(\begin{array}{l}
1 \\
0
\end{array}\right)=0, \\
& \left(\begin{array}{c}
2^{n-1}-1+2^{k}-1 \\
2^{k}-1
\end{array}\right) \bmod 2=\left(\begin{array}{l}
1 \\
0
\end{array}\right)\left(\begin{array}{l}
0 \\
0
\end{array}\right) \cdots\left(\begin{array}{l}
0 \\
0
\end{array}\right) \underbrace{\left(\begin{array}{l}
1 \\
1
\end{array}\right)} \cdots\left(\begin{array}{l}
1 \\
1
\end{array}\right)\left(\begin{array}{l}
0 \\
1
\end{array}\right)=0 .
\end{aligned}
$$

Thus, we have $w^{\left(2^{k}\right)} w^{\left(2^{n-1}-2\right)}=0, w^{\left(2^{k}\right)} w^{\left(2^{n-1}-1\right)}=0$, and $w^{\left(2^{k}-1\right)} w^{\left(2^{n-1}-1\right)}=0$. Finally, we see that $\left[e_{2^{k}-1}, \Pi o_{2^{n-1}-2}\right]=0$ for $k=1, \ldots, n-2$. Computations of the same kind show that $\left[e_{2^{k}-1}, \Pi o_{2^{n-1}-3}\right]=0$ for any $k=1, \ldots, n-2$. Therefore, $o_{2^{n-1}-2}$ and $o_{2^{n-1}-3}$ are highest weight vectors in $\mathfrak{g}_{\overline{1}}$.

Now, let us prove that there are no lowest weight vectors in $\mathfrak{g}_{\overline{1}}$. Consider the action of $e_{-1}$ on elements of $\mathfrak{g}_{\overline{1}}$. Using (6.3) and (6.4), we obtain

$$
\left[e_{-1}, \Pi o_{-1}\right]=o_{1}, \quad\left[e_{-1}, \Pi o_{k}\right]=\Pi o_{k}+\Pi o_{k-1} \quad \text { for any } k=0, \ldots, 2^{n-1}-2 .
$$

Therefore, the equation

$$
\left[e_{-1}, \sum_{-1 \leq k \leq 2^{n-1}-2} q_{k} \Pi o_{k}\right]=0
$$

for lowest weight vectors reduces to the following system of linear equations

$$
q_{-1}+q_{0}=0, \quad q_{0}+q_{1}=0, \quad \ldots, \quad q_{2^{n-1}-3}+q_{2^{n-1}-2}=0, \quad q_{2^{n-1}-2}=0 .
$$

Clearly, this system has only one solution: $q_{i}=0$ for $i=-1, \ldots, 2^{n-1}-2$. Hence, there are no lowest weight vectors in $\mathfrak{g}_{\overline{1}}$.

Observe that the highest weight vector $\Pi o_{2^{n-1}-2}$ generates the whole $\mathfrak{g}_{0}$-module $\mathfrak{g}_{\overline{1}}$ and the highest weight vector $\Pi_{2_{2}-1}-3$ generates a $\mathfrak{g}_{0}$-submodule of codimesion 1 in $\mathfrak{g}_{\overline{1}}$, as follows from (6.7).

In what follows we consider only generating functions of the form

$$
u(c)=c_{0}+\sum_{1 \leq k \leq n-1} c_{k} x^{\left(2^{k}\right)},
$$

where $c=\left(c_{0}, c_{1}, \ldots, c_{n-1}\right)$ is a set of free parameters, cf. (6.5). In what follows by "grading $c=\left(c_{0}, c_{1}, \ldots, c_{n-1}\right)$ " we mean the grading given by the corresponding $D_{u(c)}$. One should bear in mind that we call these parameters free by an abuse of the language, since they are classes modulo equivalence (6.5).

Let us consider the classification of $\mathbb{Z} / 2$-gradings of $\mathfrak{v e c t}^{(1)}(1 ; \underline{2})$ in more detail. 


\subsection{Superizations of $\mathfrak{g}=\mathfrak{v} \mathfrak{e c t}^{(1)}(1 ; \underline{2})$}

The general solution $U_{\text {lin }}$ of linear equations (1.8b), where $c_{1,1}, c_{1,2}, c_{1,3}, c_{2,1}, c_{3,1}$ are free parameters, and the general solution $U$ of equation (1.8), where $c_{0}, c_{1}$ are free parameters, and the schematic form of $U$ are as follows:

$$
U_{\text {lin }}=\left(\begin{array}{ccc}
c_{1,1} & c_{1,2} & c_{1,3} \\
c_{2,1} & 0 & c_{1,2} \\
c_{3,1} & c_{2,1} & c_{1,1}
\end{array}\right), \quad U=\left(\begin{array}{ccc}
c_{0} c_{1}+1 & c_{0} & c_{0}^{2} \\
c_{1} & 0 & c_{0} \\
c_{1}^{2} & c_{1} & c_{0} c_{1}+1
\end{array}\right), \quad\left(\begin{array}{ccc}
* & * & * \\
* & * \\
* & * & *
\end{array}\right) .
$$

This solution corresponds to the derivation $D_{u}$ with $u(c)=c_{0}+c_{1} x^{(2)}$.

Parameters and Aut $\mathfrak{g}$. We denote by (see (6.8))

$$
\mathfrak{g}_{\overline{0}}(c):=\operatorname{Ker} D_{u(c)}
$$

the even part of the corresponding $\mathbb{Z} / 2$-grading. For example, for $\mathfrak{g}=\mathfrak{v e c t}^{(1)}(1 ; \underline{3})$, by $\mathfrak{g}_{\overline{0}}\left(c_{0}, c_{1}\right)$ we mean the even part of the $\mathbb{Z} / 2$-grading $D_{u}$ where $u=c_{0}+c_{1} x^{(2)}$.

Certain automorphisms and their actions:

$$
\begin{aligned}
& \left(\begin{array}{ccc}
\frac{1}{c_{0}} & 0 & 0 \\
0 & 1 & 0 \\
\frac{c_{1}+c_{0} c_{1}}{c_{0}} & 0 & c_{0}
\end{array}\right) \quad \operatorname{maps} D_{u\left(c_{0}, c_{1}\right)} \text { to } D_{u\left(1, c_{1}\right)} \text { for all } c_{0} \neq 0, \\
& \left(\begin{array}{ccc}
1+c_{1} & 0 & 1 \\
0 & 1 & 0 \\
1 & 0 & 0
\end{array}\right) \quad \operatorname{maps} D_{u\left(1, c_{1}\right)} \text { to } D_{u(1,1)} \text { for all } c_{1} \in \mathbb{K}, \\
& \left(\begin{array}{ccc}
c_{1} & 0 & 0 \\
0 & 1 & 0 \\
0 & 0 & \frac{1}{c_{1}}
\end{array}\right) \quad \operatorname{maps} D_{u\left(0, c_{1}\right)} \text { to } D_{u(0,1)} \text { for all } c_{1} \neq 0, \\
& \left(\begin{array}{ccc}
1 & 0 & 1 \\
0 & 1 & 0 \\
0 & 0 & 1
\end{array}\right) \quad \operatorname{maps} D_{u(1,1)} \text { to } D_{u(0,1)} .
\end{aligned}
$$

Observe that the automorphism (6.10) sends the outer derivation $D_{1+x^{(2)}}$ to the inner derivation $D_{x^{(2)}}$.

The final answer: there are three inequivalent $\mathbb{Z} / 2$-gradings of $\mathfrak{v e c t}^{(1)}(1 ; \underline{2}) \simeq \mathfrak{o}^{(1)}(3)$; their $\mathfrak{g}_{0}^{\prime}$ 's are

$$
\mathfrak{g}_{\overline{0}}(0,0)=\mathbb{K} \boldsymbol{e}_{0} \quad \text { and } \quad \mathfrak{g}_{\overline{0}}(1,1)=\mathbb{K}\left(\boldsymbol{e}_{-1}+\boldsymbol{e}_{0}+\boldsymbol{e}_{1}\right)
$$

and the trivial $\mathbb{Z} / 2$-grading with $\mathfrak{g}_{\overline{0}}=\mathfrak{v e c t}^{(1)}(1 ; \underline{2})$, not two as is the case for $\mathbb{Z} / 2$-gradings of $\mathfrak{o}^{(1)}(3)=\mathfrak{o}(3)$ for any $p \neq 2$.

Lie superalgebras corresponding to the gradings (6.11).

Grading $c=(0,0)$. The minimal Lie subsuperalgebra

$$
\mathfrak{g}^{\langle 1\rangle}:=\left(\mathfrak{g}^{\langle 1\rangle}\right)_{\overline{0}} \oplus\left(\mathfrak{g}^{\langle 1\rangle}\right)_{\overline{1}}
$$

of the restricted closure $\overline{\mathfrak{g}}$ containing all elements $v^{[2]}$, where $v \in \mathfrak{g}_{\overline{1}}=\left(\mathfrak{g}^{\langle 1\rangle}\right)_{\overline{1}}$, see equation (1.5), is as follows:

$$
\begin{aligned}
& \left(\mathfrak{g}^{\langle 1\rangle}\right)_{\overline{0}}=\operatorname{Span}\left(e_{-2}=\partial^{2}, e_{0}=x \partial, e_{2}=x^{(3)} \partial\right), \\
& \left(\mathfrak{g}^{\langle 1\rangle}\right)_{\overline{1}}=\operatorname{Span}\left(o_{-1}=\partial, o_{1}=x^{(2)} \partial\right) .
\end{aligned}
$$


The squaring in $\mathfrak{s}\left(\mathfrak{g}^{\langle 1\rangle}\right)$ is given by the following formulas:

$$
o_{-1}^{2}=(\partial)^{2}=e_{-2}=\partial^{2}, \quad o_{1}^{2}=\left(x^{(2)} \partial\right)^{2}=e_{2}=x^{(3)} \partial .
$$

The commutation relations in $\left(\mathfrak{g}^{\langle 1\rangle}\right)_{\overline{0}}$ are defined by the following formulas:

$$
\left[e_{0}, e_{-2}\right]=0, \quad\left[e_{0}, e_{2}\right]=0, \quad\left[e_{-2}, e_{2}\right]=e_{0} .
$$

Observe that $\left(\mathfrak{g}^{\langle 1\rangle}\right)_{\overline{0}}$ is the Heisenberg algebra. The $\left(\mathfrak{g}^{\langle 1\rangle}\right)_{\overline{0}}$-module structure in $\left(\mathfrak{g}^{\langle 1\rangle}\right)_{\overline{1}}$ is given by the following formulas:

$$
\begin{array}{lll}
{\left[e_{-2}, o_{-1}\right]=0,} & {\left[e_{0}, o_{-1}\right]=o_{-1},} & {\left[e_{2}, o_{-1}\right]=o_{1},} \\
{\left[e_{-2}, o_{1}\right]=o_{-1},} & {\left[e_{0}, o_{1}\right]=o_{1}, \quad} & {\left[e_{2}, o_{1}\right]=0 .}
\end{array}
$$

It is easy to see that $\mathfrak{s}\left(\mathfrak{g}^{\langle 1\rangle}\right) \simeq \mathfrak{o o}_{I \Pi}^{(1)}(1 \mid 2) \simeq \mathfrak{k}(1 ; \underline{1} \mid 1)$; for the (non-obvious) definition of the $\mathfrak{k}(1 ; \underline{n} \mid 1)$, see [3]. The Lie superalgebra $\mathfrak{o o}_{I \Pi}^{(1)}(1 \mid 2)$ is given by supermatrices of the form

$$
\left(\begin{array}{ccc}
0 & b_{2} & b_{1} \\
b_{1} & a_{1} & a_{2} \\
b_{2} & a_{3} & a_{1}
\end{array}\right), \quad \text { where } a_{i}, b_{i} \in \mathbb{K} .
$$

The correspondence between the abstract and matrix representations of the elements of $\mathfrak{o} \mathfrak{o}_{I \Pi}^{(1)}(1 \mid 2)$ is as follows

$$
e_{-2}=e_{2,3}, \quad e_{0}=e_{2,2}+e_{3,3}, \quad e_{2}=e_{3,2}, \quad o_{-1}=e_{1,3}+e_{2,1}, \quad o_{1}=e_{1,2}+e_{3,1} .
$$

Grading $c=(1,1) \simeq(1,0)$. The Lie subsuperalgebra $\mathfrak{g}^{\langle 1\rangle}$, see equation $(6.12)$, is as follows

$$
\begin{aligned}
& \left(\mathfrak{g}^{\langle 1\rangle}\right)_{\overline{0}}=\operatorname{Span}\left(e_{1}=\left(1+x+x^{(2)}\right) \partial, e_{2}=\partial^{2}+(1+x) \partial, e_{3}=\partial^{2}+\left(x+x^{(3)}\right) \partial\right), \\
& \left(\mathfrak{g}^{\langle 1\rangle}\right)_{\overline{1}}=\operatorname{Span}\left(o_{1}=(1+x) \partial, o_{2}=\left(1+x^{(2)}\right) \partial\right) .
\end{aligned}
$$

The squaring in $\mathfrak{s}\left(\mathfrak{g}^{\langle 1\rangle}\right)$ is given by the following formulas:

$$
o_{1}^{2}=e_{2}, \quad o_{2}^{2}=e_{3} .
$$

The commutation relations in $\left(\mathfrak{g}^{\langle 1\rangle}\right)_{\overline{0}}$ are defined by the following formulas:

$$
\left[e_{1}, e_{2}\right]=e_{1}, \quad\left[e_{1}, e_{3}\right]=0, \quad\left[e_{2}, e_{3}\right]=e_{1} .
$$

The $\left(\mathfrak{g}^{\langle 1\rangle}\right)_{\overline{0}}$-module structure in $\left(\mathfrak{g}^{\langle 1\rangle}\right)_{\overline{1}}$ is given by the following formulas:

$$
\begin{aligned}
& {\left[e_{1}, o_{1}\right]=o_{1}+o_{2}, \quad\left[e_{2}, o_{1}\right]=0, \quad\left[e_{3}, o_{1}\right]=o_{2},} \\
& {\left[e_{1}, o_{2}\right]=o_{2}, \quad\left[e_{2}, o_{2}\right]=o_{1}+o_{2}, \quad\left[e_{3}, o_{2}\right]=0 \text {. }}
\end{aligned}
$$

Let us show that $\mathfrak{s}\left(\mathfrak{g}^{\langle 1\rangle}\right) \simeq \mathfrak{o o}_{I I}^{(1)}(1 \mid 2)$. The Lie superalgebra $\mathfrak{o o}_{I I}^{(1)}(1 \mid 2)$ consists of symmetric supermatrices with supertrace 0 . For a basis of its even part we take

$$
E_{1}=e_{1,1}+e_{2,2}, \quad E_{2}=e_{2,2}+e_{3,3}, \quad E_{3}=e_{2,3}+e_{3,2}
$$

For a basis of its odd part we take $O_{1}=e_{1,2}+e_{2,1}, O_{2}=e_{1,3}+e_{3,1}$. The commutation relations are given by the following formulas:

$$
\left[E_{1}, E_{2}\right]=0, \quad\left[E_{1}, E_{3}\right]=E_{3}, \quad\left[E_{2}, E_{3}\right]=0 .
$$


The squaring in $\mathfrak{s}\left(\mathfrak{g}^{\langle 1\rangle}\right)$ is given by the following formulas:

$$
O_{1}^{2}=E_{1}, \quad O_{2}^{2}=E_{1}+E_{2} .
$$

The $\left(\mathfrak{g}^{\langle 1\rangle}\right) \overline{0}^{- \text {module structure is as follows }}$

$$
\left[E_{2}, O_{1}\right]=O_{1}, \quad\left[E_{2}, O_{2}\right]=O_{2} .
$$

The multiplication by elements $E_{1}$ and $E_{3}$ is given by the following formulas:

$$
\left[E_{1}, O_{1}\right]=0, \quad\left[E_{1}, O_{2}\right]=O_{2}, \quad\left[E_{3}, O_{1}\right]=O_{2}, \quad\left[E_{3}, O_{2}\right]=O_{1} .
$$

The isomorphism between $\mathfrak{o o}_{I I}^{(1)}(1 \mid 2)$ and $\mathfrak{g}^{\langle 1\rangle}$ is given by the following formulas:

$$
e_{1}=E_{3}, \quad e_{2}=E_{1}, \quad e_{3}=E_{2}+E_{3}, \quad o_{1}=O_{1}, \quad o_{2}=O_{1}+O_{2} .
$$

\section{Remark 6.6.}

1) Observe that the even parts of both superizations are solvable Lie algebras, but the corresponding Lie superalgebras are simple. For more details and examples of this phenomenon indigenous to $p=2$, see Shchepochkina's comment (the last section) in [6].

2) Observe that due to Theorem 6.2 the Lie superalgebra $\mathfrak{o o}_{I I}^{(1)}(1 \mid 2)$ is a filtered deform of the Lie superalgebra $\mathfrak{o o}_{I \Pi}^{(1)}(1 \mid 2)$.

\subsection{Superizations corresponding to inner tori}

Consider a superization of $\mathfrak{v e c t}^{(1)}(1 ; \underline{n})$ given by a generating function $u$ such that $u(0)=0$. For such a function $u$, the derivation $D_{u}$ spans an inner torus. According to [29], the derivation $D_{u}$ is conjugate to the derivation $D_{f}$ in $\mathfrak{v e c t}^{(1)}(1 ; \underline{n})$, where $f=\sum_{1 \leq i \leq n-1} c_{i} x^{\left(2^{i}\right)}$ and the $c_{i} x^{\left(2^{i}\right)}$ are the corresponding terms of $u$, so the superizations corresponding to $D_{u}$ and $D_{f}$ are isomorphic. For this reason, in the rest of this section we consider only generating functions of the form $\sum_{1 \leq i \leq n-1} c_{i} x^{\left(2^{i}\right)}$. This gives us an $(n-1)$-parametric family of $\mathbb{Z} / 2$-gradings.

Conjecture 6.7. Let $u_{1}=\sum_{1 \leq k \leq n-1} c_{k} x^{\left(2^{k}\right)}$ and $u_{2}=\sum_{1 \leq k \leq n-1} b_{k} x^{\left(2^{k}\right)}$ be generating functions, then the superizations corresponding to $D_{u_{1}}$ and $D_{u_{2}}$ are isomorphic if and only if there exists $\varepsilon \in \mathbb{K}^{\times}$such that $c_{k}=\varepsilon^{2^{k}-1} b_{k}$ for all $k=1, \ldots, n-1$.

The following is a sketch of a proof of this conjecture, including a complete computer-aided proof for $2 \leq n \leq 6$.

First of all, if there is an $\varepsilon \neq 0$ such that $c_{k}=\varepsilon^{2^{k}-1} b_{k}$ for all $k=1, \ldots, n-1$, then $D_{u_{1}}$ and $D_{u_{2}}$ are conjugate by the automorphism of $\mathfrak{v e c t} \mathfrak{c t}^{(1)}(1 ; \underline{n})$ given by $x^{(m)} \partial \mapsto \varepsilon^{m-1} x^{(m)} \partial$ (this automorphism is generated by the automorphism of $\mathcal{O}(1 ; \underline{n})$ given by $\left.x^{(m)} \mapsto \varepsilon^{m} x^{(m)}\right)$, and therefore the corresponding superizations are isomorphic.

By Theorem 6.2, the superization given by $u_{1}$ is isomorphic to a deform of $\mathfrak{k}(1 ; \underline{n-1} \mid 1)$. Consider the even part of such a deform. According to (6.6), it contains a commutative subalgebra of codimension 1 , which is the 0 -even part of $\mathfrak{v e c t}(1 ; \underline{n})$. The element $\partial^{2}$ acts on this commutative subalgebra, and its action is given by

$$
v \partial \mapsto\left(1+u \partial^{2} u\right) \partial^{2} v \partial \quad \text { for any } 0 \text {-odd } v \in \mathcal{O}(1 ; \underline{n}) .
$$


Conjecture 6.8 (proved by computer for $n=2,3,4,5,6)$. The characteristic polynomial of the linear operator $(6.14)$ on the 0 -even part of $\mathfrak{v e c t}(1 ; \underline{n})$ for $n \geq 2$ is defined by the formula

$$
\lambda^{2^{n-1}}+\sum_{0 \leq k \leq n-2} c_{n-1-k}^{2^{k+1}} \lambda^{2^{k}} .
$$

For two deforms given by $u_{1}$ and $u_{2}$ to be isomorphic to each other, their even parts have to be isomorphic, which means that the two actions of $\partial^{2}$ on the 0 -even part of $\mathfrak{v e c t}(1 ; \underline{n})$ have to be conjugate (similar) up to a non-zero scalar factor, i.e., if $A$ and $A^{\prime}$ are such operators, then $A^{\prime}=\alpha M A M^{-1}$ for some non-zero $\alpha \in \mathbb{K}$ and an invertible linear map $M$. If two operators are conjugate up to a scalar multiple $\alpha$, the roots of their characteristic polynomials differ by the same multiple, so if the dimension of the space they act on is $d$ (in our case, $d=2^{n-1}$ ) and one polynomial is equal to $\sum_{0 \leq i \leq d} z_{i} \lambda^{i}$, where $z_{i} \in \mathbb{K}$ and $z_{d}=1$, then the other polynomial would have the form $\sum_{0 \leq i \leq d} z_{i} \alpha^{d-i} \lambda^{i}$. So, if Conjecture 6.8 is correct, then for the superizations given by $u_{1}$ and $u_{2}$ to be isomorphic, there must exist a non-zero $\alpha \in \mathbb{K}$ such that

$$
\lambda^{2^{n-1}}+\sum_{0 \leq k \leq n-2} c_{n-1-k}^{2^{k+1}} \lambda^{2^{k}}=\lambda^{2^{n-1}}+\sum_{0 \leq k \leq n-2} \alpha^{2^{n-1}-2^{k}} b_{n-1-k}^{2^{k+1}} \lambda^{2^{k}},
$$

or, equivalently, $c_{k}=\varepsilon^{2^{k}-1} b_{k}$, where $\varepsilon=\sqrt{\alpha}$, for all $k=1, \ldots, n-1$. Thus, Conjecture 6.7 follows from Conjecture 6.8.

So, assuming that Conjecture 6.8 is true, the set of equivalence classes of superizations of $\mathfrak{v e c t} \mathfrak{t}^{(1)}(1 ; \underline{n})$ corresponding to generating functions $u$ such that $u(0)=0$ consist of the following two types:

A) the superization corresponding to $u=0$, which is isomorphic to $\mathfrak{k}(1 ; \underline{n-1} \mid 1)$,

B) an $(n-2)$-parametric family of its pairwise non-isomorphic deforms. Note, though, that it is not a result of $(n-2)$-parametric deformation of $\mathfrak{k}(1 ; \underline{n-1 \mid} \mid 1)$; to obtain all these deforms, an $(n-1)$-parametric deformation is needed, but the deforms obtained from some sets of parameters are isomorphic: parameters $\left(c_{1}, \ldots, c_{n-1}\right)$ and $\left(b_{1}, \ldots, b_{n-1}\right)$ produce isomorphic deformations if and only if there exists an $\varepsilon \in \mathbb{K}^{\times}$such that $c_{k}=\varepsilon^{2^{k}-1} b_{k}$ for all $k \in 1, \ldots, n-1$.

\subsection{Superizations of $\mathfrak{v e c t}^{(1)}(1 ; \underline{3})$}

Let $\mathfrak{g}=\mathfrak{v e c t}^{(1)}(1 ; \underline{3})$.

Grading $c=(0,0,0)$. Recall our notation (6.9). The Lie subsuperalgebra $\mathfrak{g}^{\langle 1\rangle}$, see equation (6.12), is as follows:

$$
\begin{aligned}
& \left(\mathfrak{g}^{\langle 1\rangle}\right)_{\overline{0}}=\operatorname{Span}\left(e_{-2}=\partial^{2}, e_{0}=x \partial, e_{2}=x^{(3)} \partial, e_{4}=x^{(5)} \partial, e_{6}=x^{(7)} \partial\right) \\
& \left(\mathfrak{g}^{\langle 1\rangle}\right)_{\overline{1}}=\operatorname{Span}\left(o_{-1}=\partial, o_{1}=x^{(2)} \partial, o_{3}=x^{(4)} \partial, o_{5}=x^{(6)} \partial\right)
\end{aligned}
$$

The squaring in $\mathfrak{s}\left(\mathfrak{g}^{\langle 1\rangle}\right)$ is given by the following formulas:

$$
o_{-1}^{2}=e_{-2}, \quad o_{1}^{2}=e_{2}, \quad o_{3}^{2}=e_{6}, \quad o_{5}^{2}=0 .
$$

The nonzero commutation relations in $\left(\mathfrak{g}^{\langle 1\rangle}\right)_{\overline{0}}$ are given by the following formulas:

$$
\left[e_{2}, e_{-2}\right]=e_{0}, \quad\left[e_{4}, e_{-2}\right]=e_{2}, \quad\left[e_{-2}, e_{6}\right]=e_{4} .
$$


The $\left(\mathfrak{g}^{\langle 1\rangle}\right)_{\overline{0}}$-module structure in $\left(\mathfrak{g}^{\langle 1\rangle}\right)_{\overline{1}}$ is given by the following formulas:

$$
\begin{aligned}
& {\left[e_{-2}, o_{-1}\right]=0, \quad\left[e_{0}, o_{-1}\right]=o_{-1}, \quad\left[e_{2}, o_{-1}\right]=o_{1}, \quad\left[e_{4}, o_{-1}\right]=o_{3}, \quad\left[e_{6}, o_{-1}\right]=o_{5},} \\
& {\left[e_{-2}, o_{1}\right]=o_{-1}, \quad\left[e_{0}, o_{1}\right]=o_{1}, \quad\left[e_{2}, o_{1}\right]=0, \quad\left[e_{4}, o_{1}\right]=o_{5}, \quad\left[e_{6}, o_{1}\right]=0,} \\
& {\left[e_{-2}, o_{3}\right]=o_{1}, \quad\left[e_{0}, o_{3}\right]=o_{3}, \quad\left[e_{2}, o_{3}\right]=o_{5}, \quad\left[e_{4}, o_{3}\right]=0, \quad\left[e_{6}, o_{3}\right]=0,} \\
& {\left[e_{-2}, o_{5}\right]=o_{3}, \quad\left[e_{0}, o_{5}\right]=o_{5}, \quad\left[e_{2}, o_{5}\right]=0, \quad\left[e_{4}, o_{5}\right]=0, \quad\left[e_{6}, o_{5}\right]=0 .}
\end{aligned}
$$

Let $L_{k}$ and $L^{(k)}$ be given by the formulas

$$
L_{0}=L^{0}=\left(\mathfrak{g}^{\langle 1\rangle}\right)_{\overline{0}}, \quad L_{k}=\left[L_{0}, L_{k-1}\right], \quad L^{(k)}=\left[L^{(k-1)}, L^{(k-1)}\right] \quad \text { for } k>0 .
$$

We have

$$
L_{1}=\operatorname{Span}\left(e_{0}, e_{2}, e_{4}\right), \quad L_{2}=\operatorname{Span}\left(e_{0}, e_{2}\right), \quad L_{3}=\mathbb{K} e_{0}, \quad L_{4}=0 .
$$

Grading $c=(0,1,0)$. The Lie subsuperalgebra $\mathfrak{g}^{\langle 1\rangle}$, see equation $(6.12)$, is as follows:

$$
\begin{aligned}
\left(\mathfrak{g}^{\langle 1\rangle}\right)_{\overline{0}}=\operatorname{Span}\left(e_{1}\right. & =\left(x+x^{(2)}\right) \partial, e_{2}=x^{(3)} \partial, e_{3}=\left(x^{(5)}+x^{(6)}\right) \partial, \\
e_{4} & \left.=\partial^{2}+(1+x) \partial, e_{5}=x^{(7)} \partial\right), \\
\left(\mathfrak{g}^{\langle 1\rangle}\right)_{\overline{1}}=\operatorname{Span}\left(o_{1}\right. & \left.=(1+x) \partial, o_{2}=x^{(2)} \partial, o_{3}=\left(x^{(4)}+x^{(5)}\right) \partial, o_{4}=x^{(6)} \partial\right) .
\end{aligned}
$$

The squaring in $\mathfrak{s}\left(\mathfrak{g}^{\langle 1\rangle}\right)$ is given by the following formulas:

$$
o_{1}^{2}=e_{4}, \quad o_{2}^{2}=e_{2}, \quad o_{3}^{2}=e_{5}, \quad o_{4}^{2}=0 .
$$

The non-zero commutation relations in $\left(\mathfrak{g}^{\langle 1\rangle}\right)_{\overline{0}}$ are given by the following formulas:

$$
\left[e_{1}, e_{4}\right]=e_{1}, \quad\left[e_{2}, e_{4}\right]=e_{1}, \quad\left[e_{3}, e_{4}\right]=e_{2}+e_{3}, \quad\left[e_{4}, e_{5}\right]=e_{3} .
$$

The $\left(\mathfrak{g}^{\langle 1\rangle}\right)_{\overline{0}}$-module structure in $\left(\mathfrak{g}^{\langle 1\rangle}\right)_{\overline{1}}$ is given by the following formulas:

$$
\begin{aligned}
& {\left[e_{1}, o_{1}\right]=o_{1}+o_{2}, \quad\left[e_{2}, o_{1}\right]=o_{2}, \quad\left[e_{3}, o_{1}\right]=o_{3}+o_{4}, \quad\left[e_{4}, o_{1}\right]=0, \quad\left[e_{5}, o_{1}\right]=o_{4},} \\
& {\left[e_{1}, o_{2}\right]=o_{2}, \quad\left[e_{2}, o_{2}\right]=0, \quad\left[e_{3}, o_{2}\right]=o_{4}, \quad\left[e_{4}, o_{2}\right]=o_{1}+o_{2}, \quad\left[e_{5}, o_{2}\right]=0,} \\
& {\left[e_{1}, o_{3}\right]=o_{3}+o_{4}, \quad\left[e_{2}, o_{3}\right]=o_{4}, \quad\left[e_{3}, o_{3}\right]=0, \quad\left[e_{4}, o_{3}\right]=o_{2}, \quad\left[e_{5}, o_{3}\right]=0,} \\
& {\left[e_{1}, o_{4}\right]=o_{4}, \quad\left[e_{2}, o_{4}\right]=0, \quad\left[e_{3}, o_{4}\right]=0, \quad\left[e_{4}, o_{4}\right]=o_{3}+o_{4}, \quad\left[e_{5}, o_{4}\right]=0 \text {. }}
\end{aligned}
$$

Grading $c=(0,1, \beta)$, where $\beta \neq 0$. The Lie subsuperalgebra $\mathfrak{g}^{\langle 1\rangle}$, see equation (6.12), is as follows:

$$
\begin{aligned}
\left(\mathfrak{g}^{\langle 1\rangle}\right)_{\overline{0}}=\operatorname{Span}\left(e_{1}\right. & =\left(x+x^{(2)}+\beta x^{(4)}\right) \partial, e_{2}=\left(x^{(3)}+\beta x^{(5)}\right) \partial, e_{3}=\left(x^{(5)}+x^{(6)}\right) \partial, \\
e_{4} & \left.=\partial^{2}+\left(1+x+\beta x^{(2)}\right) \partial, e_{5}=\left(x^{(3)}+\beta x^{(5)}+\beta^{2} x^{(7)}\right) \partial\right), \\
\left(\mathfrak{g}^{\langle 1\rangle}\right)_{\overline{1}}=\operatorname{Span}\left(o_{1}\right. & =\left(1+x+\beta x^{(3)}\right) \partial, o_{2}=\left(x^{(2)}+\beta x^{(4)}\right) \partial, \\
o_{3} & \left.=\left(x^{(4)}+x^{(5)}\right) \partial, o_{4}=x^{(6)} \partial\right) .
\end{aligned}
$$

The squaring in $\mathfrak{s}\left(\mathfrak{g}^{\langle 1\rangle}\right)$ is given by the following formulas:

$$
o_{1}^{2}=e_{4}, \quad o_{2}^{2}=e_{5}, \quad o_{3}^{2}=\frac{1}{\beta^{2}} e_{2}+\frac{1}{\beta^{2}} e_{5}, \quad o_{4}^{2}=0 .
$$

The non-zero commutation relations in $\left(\mathfrak{g}^{\langle 1\rangle}\right)_{\overline{0}}$ are defined by the following formulas:

$$
\begin{aligned}
& {\left[e_{1}, e_{4}\right]=e_{1}+\beta e_{2},} \\
& {\left[e_{2}, e_{4}\right]=e_{1}+\beta e_{2}+\beta^{2} e_{3}, \quad\left[e_{3}, e_{4}\right]=e_{2}+(1+\beta) e_{3}, \quad\left[e_{4}, e_{5}\right]=e_{1}+\beta e_{2} .}
\end{aligned}
$$


The $\left(\mathfrak{g}^{\langle 1\rangle}\right)_{\overline{0}}$-module structure in $\left(\mathfrak{g}^{\langle 1\rangle}\right)_{\overline{1}}$ is given by the following formulas:

$$
\begin{aligned}
& {\left[e_{1}, o_{1}\right]=o_{1}+o_{2}+\beta^{2} o_{4}, \quad\left[e_{2}, o_{1}\right]=o_{2}, \quad\left[e_{3}, o_{1}\right]=o_{3}+o_{4}, \quad\left[e_{4}, o_{1}\right]=0,} \\
& {\left[e_{1}, o_{2}\right]=o_{2}, \quad\left[e_{2}, o_{2}\right]=0, \quad\left[e_{3}, o_{2}\right]=o_{4},} \\
& {\left[e_{4}, o_{2}\right]=o_{1}+(1+\beta) o_{2}+\beta^{2} o_{3},} \\
& {\left[e_{1}, o_{3}\right]=o_{3}+o_{4}, \quad\left[e_{2}, o_{3}\right]=o_{4}, \quad\left[e_{3}, o_{3}\right]=0,} \\
& {\left[e_{4}, o_{3}\right]=o_{2}+\beta\left(o_{3}+o_{4}\right) \text {, }} \\
& {\left[e_{1}, o_{4}\right]=o_{4}, \quad\left[e_{2}, o_{4}\right]=0, \quad\left[e_{3}, o_{4}\right]=0, \quad\left[e_{4}, o_{4}\right]=o_{3}+o_{4},} \\
& {\left[e_{5}, o_{1}\right]=o_{2}+\beta^{2} o_{4}, \quad\left[e_{5}, o_{2}\right]=0, \quad\left[e_{5}, o_{3}\right]=o_{4}, \quad\left[e_{5}, o_{4}\right]=0 .}
\end{aligned}
$$

Grading $c=(0,0,1)$. The Lie subsuperalgebra $\mathfrak{g}^{\langle 1\rangle}$, see equation $(6.12)$, is as follows:

$$
\begin{aligned}
\left(\mathfrak{g}^{\langle 1\rangle}\right)_{\overline{0}}=\operatorname{Span}\left(e_{1}\right. & =\left(x+x^{(4)}\right) \partial, e_{2}=x^{(5)} \partial \\
e_{3} & \left.=\left(x^{(3)}+x^{(6)}\right) \partial, e_{4}=\partial^{2}+x^{(2)} \partial, e_{5}=x^{(7)} \partial\right), \\
\left(\mathfrak{g}^{\langle 1\rangle}\right)_{\overline{1}}=\operatorname{Span}\left(o_{1}\right. & \left.=\left(1+x^{(3)}\right) \partial, o_{2}=x^{(4)} \partial, o_{3}=\left(x^{(2)}+x^{(5)}\right) \partial, o_{4}=x^{(6)} \partial\right) .
\end{aligned}
$$

The squaring in $\mathfrak{s}\left(\mathfrak{g}^{\langle 1\rangle}\right)$ is given by the following formulas:

$$
o_{1}^{2}=e_{4}, \quad o_{2}^{2}=e_{5}, \quad o_{3}^{2}=e_{3}, \quad o_{4}^{2}=0 .
$$

The non-zero commutation relations in $\left(\mathfrak{g}^{\langle 1\rangle}\right)_{\overline{0}}$ are given by the following formulas:

$$
\left[e_{1}, e_{4}\right]=e_{2}, \quad\left[e_{2}, e_{4}\right]=e_{3}, \quad\left[e_{3}, e_{4}\right]=e_{1}, \quad\left[e_{4}, e_{5}\right]=e_{2} .
$$

The $\left(\mathfrak{g}^{\langle 1\rangle}\right)_{\overline{0}}$-module structure in $\left(\mathfrak{g}^{\langle 1\rangle}\right)_{\overline{1}}$ is given by the following formulas:

$$
\begin{aligned}
& {\left[e_{1}, o_{1}\right]=o_{1}+o_{4}, \quad\left[e_{2}, o_{1}\right]=o_{2}, \quad\left[e_{3}, o_{1}\right]=o_{3}, \quad\left[e_{4}, o_{1}\right]=0, \quad\left[e_{5}, o_{1}\right]=o_{4},} \\
& {\left[e_{1}, o_{2}\right]=o_{2}, \quad\left[e_{2}, o_{2}\right]=0, \quad\left[e_{3}, o_{2}\right]=o_{4}, \quad\left[e_{4}, o_{2}\right]=o_{3}, \quad\left[e_{5}, o_{2}\right]=0,} \\
& {\left[e_{1}, o_{3}\right]=o_{3}, \quad\left[e_{2}, o_{3}\right]=o_{4}, \quad\left[e_{3}, o_{3}\right]=0, \quad\left[e_{4}, o_{3}\right]=o_{1}+o_{4}, \quad\left[e_{5}, o_{3}\right]=0,} \\
& {\left[e_{1}, o_{4}\right]=o_{4}, \quad\left[e_{2}, o_{4}\right]=0, \quad\left[e_{3}, o_{4}\right]=0, \quad\left[e_{4}, o_{4}\right]=o_{2}, \quad\left[e_{5}, o_{4}\right]=0 .}
\end{aligned}
$$

Grading $c=(1,0,0)$. The Lie subsuperalgebra $\mathfrak{g}^{\langle 1\rangle}$, see equation $(6.12)$, is as follows:

$$
\begin{gathered}
\left(\mathfrak{g}^{\langle 1\rangle}\right)_{\overline{0}}=\operatorname{Span}\left(e_{-2}=\partial^{2}+(1+x) \partial, e_{-1}=(1+x) \partial, e_{0}=\left(x+x^{(2)}+x^{(3)}\right) \partial,\right. \\
\left.e_{1}=\left(x^{(2)}+x^{(4)}+x^{(5)}\right) \partial, e_{2}=\left(x^{(3)}+x^{(5)}+x^{(6)}+x^{(7)}\right) \partial\right), \\
\left(\mathfrak{g}^{\langle 1\rangle}\right)_{\overline{1}}=\operatorname{Span}\left(o_{1}=\partial, o_{2}=\left(x+x^{(2)}\right) \partial,\right. \\
\left.o_{3}=\left(x^{(2)}+x^{(3)}+x^{(4)}\right) \partial, o_{4}=\left(x^{(3)}+x^{(5)}+x^{(6)}\right) \partial\right) .
\end{gathered}
$$

The squaring in $\mathfrak{s}\left(\mathfrak{g}^{\langle 1\rangle}\right)$ is given by the following formulas:

$$
o_{1}^{2}=e_{-2}, \quad o_{2}^{2}=e_{0}, \quad o_{3}^{2}=e_{2}, \quad o_{4}^{2}=0 .
$$

The non-zero commutation relations in $\left(\mathfrak{g}^{\langle 1\rangle}\right)_{\overline{0}} \simeq(\mathfrak{v e c t}(1 ; \underline{3}))^{\langle 1\rangle}$ are given by the following formulas:

$$
\left[e_{-1}, e_{0}\right]=e_{-1}, \quad\left[e_{-1}, e_{1}\right]=e_{0}, \quad\left[e_{-1}, e_{2}\right]=e_{1}, \quad\left[e_{1}, e_{-2}\right]=e_{-1}, \quad\left[e_{0}, e_{1}\right]=e_{1} .
$$

The $\left(\mathfrak{g}^{\langle 1\rangle}\right)_{\overline{0}}$-module structure in $\left(\mathfrak{g}^{\langle 1\rangle}\right)_{\overline{1}}$ is given by the following formulas:

$$
\left[e_{-1}, o_{1}\right]=o_{1}, \quad\left[e_{0}, o_{1}\right]=o_{1}+o_{2}, \quad\left[e_{1}, o_{1}\right]=o_{2}+o_{3}, \quad\left[e_{2}, o_{1}\right]=o_{3}+o_{4},
$$




$$
\begin{aligned}
& {\left[e_{-2}, o_{1}\right]=o_{1}} \\
& {\left[e_{-1}, o_{2}\right]=o_{1}+o_{2}, \quad\left[e_{0}, o_{2}\right]=0, \quad\left[e_{1}, o_{2}\right]=o_{3}+o_{4}, \quad\left[e_{2}, o_{2}\right]=0,} \\
& {\left[e_{-2}, o_{2}\right]=o_{2} \text {, }} \\
& {\left[e_{-1}, o_{3}\right]=o_{2}+o_{3}, \quad\left[e_{0}, o_{3}\right]=o_{3}+o_{4}, \quad\left[e_{1}, o_{3}\right]=0, \quad\left[e_{2}, o_{3}\right]=0,} \\
& {\left[e_{-2}, o_{3}\right]=o_{1}+o_{3} \text {, }} \\
& {\left[e_{-1}, o_{4}\right]=o_{3}+o_{4}, \quad\left[e_{0}, o_{4}\right]=0, \quad\left[e_{1}, o_{4}\right]=0, \quad\left[e_{2}, o_{4}\right]=0,} \\
& {\left[e_{-2}, o_{4}\right]=o_{2}+o_{4} \text {. }}
\end{aligned}
$$

We have

$$
\begin{aligned}
& {\left[\left(\mathfrak{g}^{\langle 1\rangle}\right)_{\overline{0}},\left(\mathfrak{g}^{\langle 1\rangle}\right)_{\overline{0}}\right]=\operatorname{Span}\left(e_{-1}, e_{0}, e_{1}\right)} \\
& {\left[\left(\mathfrak{g}^{\langle 1\rangle}\right)_{\overline{0}},\left[\left(\mathfrak{g}^{\langle 1\rangle}\right)_{\overline{0}},\left(\mathfrak{g}^{\langle 1\rangle}\right)_{\overline{0}}\right]\right]=\left[\left(\mathfrak{g}^{\langle 1\rangle}\right)_{\overline{0}},\left(\mathfrak{g}^{\langle 1\rangle}\right)_{\overline{0}}\right]}
\end{aligned}
$$

The ideal $\left[\left(\mathfrak{g}^{\langle 1\rangle}\right)_{\overline{0}},\left(\mathfrak{g}^{\langle 1\rangle}\right)_{\overline{0}}\right]$ is isomorphic to $\mathfrak{o}^{(1)}(3)$. The $\mathfrak{o}^{(1)}(3)$-module $\left(\mathfrak{g}^{\langle 1\rangle}\right)_{\overline{1}}$ is irreducible, it

\begin{tabular}{|c|c|c|c|c|}
\hline \multirow{5}{*}{$\mathfrak{v e c t} \mathfrak{t}^{(1)}(1 ; \underline{3})$} & $\left(\mathfrak{g}^{\langle 1\rangle}\right)_{\overline{0}}$ & $\operatorname{dim} L_{k}$ & $\operatorname{dim} L^{(k)}$ & parameters \\
\hline & $\mathfrak{o}(3) / \mathfrak{c} \simeq \mathfrak{v}_{3}$ & 3 & 3 & $(1 a b)$ \\
\hline & solv & $3,2,1,0$ & 3,0 & $(000)$ \\
\hline & solv & 3,2 & 3,0 & $(010),(010),(0 \alpha 0)$, where $\alpha \neq 0$ \\
\hline & solv & 3 & 3,0 & $(001),(011),(0 \alpha \beta)$, where $\beta \neq 0$ \\
\hline
\end{tabular}
has no lowest weight vectors and has highest vectors $o_{3}$ and $o_{4}$, cf. [11].

\subsection{Summary of computer-aided experiments}

Let $L_{k}$ and $L^{(k)}$ be given by equation (6.15); in tables below, $k=1,2, \ldots$ up to first stable term. Theorem 6.2 implies the following fact:

$$
\begin{aligned}
& \operatorname{sdim} \mathfrak{s}\left(\mathfrak{g}, D_{u(c)}\right)=\left(2^{n-1}+1 \mid 2^{n-1}\right) \quad \text { and } \\
& \operatorname{dim}\left[\left(\mathfrak{g}^{\langle 1\rangle}\right)_{\overline{0}},\left(\mathfrak{g}^{\langle 1\rangle}\right)_{\overline{0}}\right]=2^{n-1}-1 .
\end{aligned}
$$

Recall that $\mathfrak{v}_{n}$ is defined by (6.1); the symbol "solv" below means that the corresponding Lie algebra is solvable, see Corollary 6.4. Computer-aided experiments show that

\begin{tabular}{|l|l|l|l|l|}
\hline $\mathfrak{v e c t}^{(1)}(1 ; \underline{2})$ & $\left(\mathfrak{g}^{\langle 1\rangle}\right)_{\overline{0}}$ & $\operatorname{dim} L_{k}$ & $\operatorname{dim} L^{(k)}$ & parameters \\
\cline { 2 - 5 } & Heisenberg & 1,0 & 1,0 & $(00)$ \\
\hline solv, see $(6.13)$ & 1 & 1,0 & $(01),(10),(11)$ \\
\hline
\end{tabular}

\begin{tabular}{|l|l|l|l|l|}
\hline $\mathfrak{g}_{\overline{0}}^{\langle 1\rangle}$ & $\operatorname{dim} L_{k}$ & $\operatorname{dim} L^{(k)}$ & parameters \\
\hline $\mathfrak{v}_{4}$ & 7 & 7 & $(1 a b c)$ \\
\hline $\operatorname{solv}$ & $7,6,4)$ & $7,6,4,3,2,1,0$ & 7,0 & $(0000)$ \\
\cline { 2 - 5 } & $\operatorname{solv}$ & $7,6,4$ & 7,0 & $(0100)$ \\
\hline $\operatorname{solv}$ & 7,6 & 7,0 & $(0010),(0110)$ \\
\hline $\operatorname{solv}$ & 7 & 7,0 & $(0001),(0011),(0101),(0111)$ \\
\hline
\end{tabular}




\begin{tabular}{|l|l|l|l|l|}
\hline \multirow{2}{*}{$\mathfrak{g}_{\overline{0}}^{\langle 1\rangle}$} & $\operatorname{dim} L_{k}$ & $\operatorname{dim} L^{(k)}$ & parameters \\
\hline $\mathfrak{v}_{5}$ & 15 & 15 & $(1 a b c d)$ \\
\cline { 2 - 4 } & solv & $15,14,13,12,11,10,9,8$, & 15,0 & $(00000)$ \\
$7,6,5,4,3,2,1,0$ & & & \\
\cline { 2 - 5 } & solv & $15,14,13,12,11,10,9,8$ & 15,0 & $(01000)$ \\
\cline { 2 - 5 } & solv & $15,14,13,12$ & 15,0 & $(00100),(01100)$ \\
\cline { 2 - 4 } & solv & 15,14 & 15,0 & $(00010),(00110),(01010),(01110)$ \\
\hline solv & 15 & 15,0 & $(00001),(00011),(00101),(00111)$, \\
& & & $(01001),(01011),(01101),(01111)$ \\
\hline
\end{tabular}

Let $\mathfrak{i}$ be the last term of the sequence $L_{k}$, see (6.15), for the even part $\mathfrak{g}_{\overline{0}}$ of the corresponding Lie superalgebra $\mathfrak{g}$. Set

$$
c(i):=(0 \ldots 010 \ldots 0) \quad \text { with a } 1 \text { in the }(i+1) \text { st slot. }
$$

Conjecture 6.9. Grading $c(k)$ with the corresponding generating function $u=x^{\left(2^{k}\right)}$, where $k=1, \ldots, n-1$, yields the Lie superalgebras such that $\operatorname{dim} \mathfrak{i}=2^{n-1}-2^{n-1-k}$.

The following two conjectures concern $\mathfrak{v e c t}^{(1)}(1 ; \underline{n})$, where $n \geq 3$.

Conjecture 6.10. Let $u=\sum_{1 \leq i \leq n-1} c_{i} x^{\left(2^{i}\right)}$, i.e., $c_{0}=0$. If all $c_{i}$ are equal to 0 , then let $k$ be 0 ; otherwise, let $k$ be the maximal number such that $c_{k} \neq 0$. Then the grading given by $D_{u}$ yields the Lie superalgebras such that $\operatorname{dim} \mathfrak{i}=2^{n-1}-2^{n-1-k}$.

\subsection{The derivations of $\mathfrak{v e c t} \mathfrak{t}^{(1)}(1 ; \underline{n})$}

The result of this subsection is probably known, but we'd like to draw attention to Sierpiński sieves here. Observe that solutions of the linear equations $(1.8 b)$, i.e., derivations of $\mathfrak{v e c t}^{(1)}(1 ; \underline{n})$, form the Sierpiński sieve of order $\underline{n}$ under the main diagonal, i.e., for $i \geq j$, we have

$$
c_{i, j}=\left(\begin{array}{c}
i \\
j-1
\end{array}\right) c_{i-j+1,1}, \quad \text { which corresponds to the derivation } \operatorname{ad}_{x^{(i-j+1)} \partial},
$$

and for $i<j$ we have nonzero diagonals with parameters $c_{1,2^{k}+1}$, where $k=0, \ldots, n-1$, which correspond to the derivations $\operatorname{ad}_{\partial^{2}}$, e.g.,

$$
\begin{aligned}
& c_{1, j}=0 \quad \text { if } j \neq 2^{k}+1, \text { where } k \text { is a non-negative integer, } \\
& c_{i, j}=c_{i-1, j-1} \quad \text { for } i>1 .
\end{aligned}
$$

Namely, for $\underline{n}=3$, we have the following solution of linear equations (1.8b), and its schematic form (here $*$ represents any nonzero entry and the empty entries represent zeros):

$$
U_{\text {lin }}=\left(\begin{array}{ccccccc}
c_{1,1} & c_{1,2} & c_{1,3} & 0 & c_{1,5} & 0 & 0 \\
c_{2,1} & 0 & c_{1,2} & c_{1,3} & 0 & c_{1,5} & 0 \\
c_{3,1} & c_{2,1} & c_{1,1} & c_{1,2} & c_{1,3} & 0 & c_{1,5} \\
c_{4,1} & 0 & 0 & 0 & c_{1,2} & c_{1,3} & 0 \\
c_{5,1} & c_{4,1} & 0 & 0 & c_{1,1} & c_{1,2} & c_{1,3} \\
c_{6,1} & 0 & c_{4,1} & 0 & c_{2,1} & 0 & c_{1,2} \\
c_{7,1} & c_{6,1} & c_{5,1} & c_{4,1} & c_{3,1} & c_{2,1} & c_{1,1}
\end{array}\right), \quad\left(\begin{array}{ccccccc}
* & * & * & & * & & \\
* & & * & * & & * & \\
* & * & * & * & * & & * \\
* & & & & * & * & \\
* & * & & & * & * & * \\
* & & * & & * & & * \\
* & * & * & * & * & * & *
\end{array}\right) .
$$


For $\underline{n}=4$, we have the following schematic form of the solution of linear equations (1.8b):

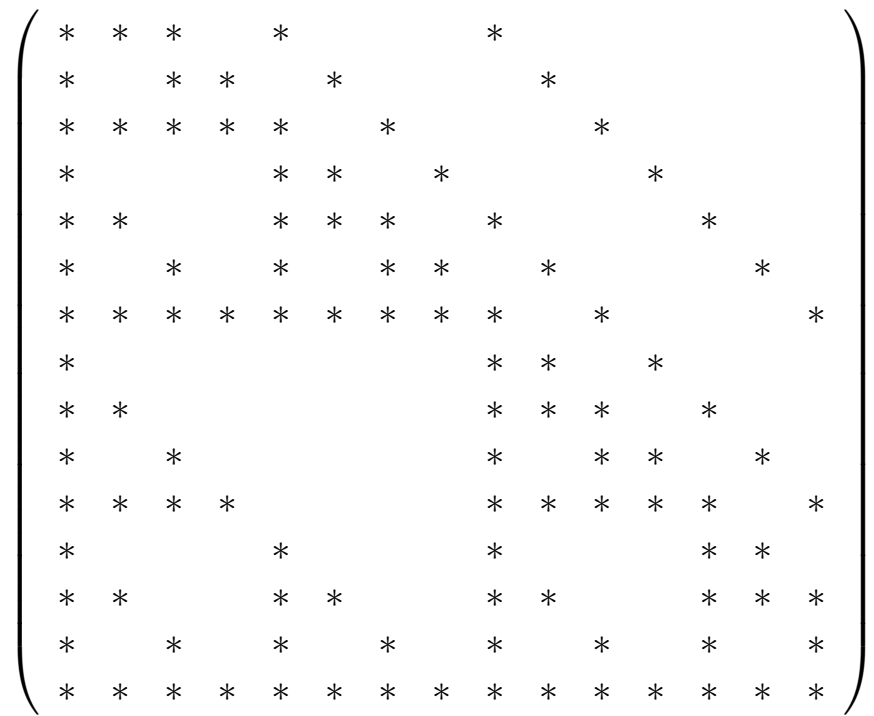

\section{A Necessary proofs from [2]}

Statement A.1 ([2, Statement 3.8.1.a]). For $n=3$ or $n \geq 5$, the algebra $\mathfrak{d e r} \mathfrak{o}_{I}^{(1)}(n)$ can be identified with $\mathfrak{o}_{I}(n) / \mathfrak{c}$ in the sense that for any $D \in \mathfrak{d e r} \mathfrak{o}_{I}^{(1)}(n)$, there is $A_{D} \in \mathfrak{o}_{I}(n)$ such that $D$ coincides with the restriction of $\operatorname{ad}_{A_{D}}$ to $\mathfrak{o}_{I}^{(1)}(n)$; for a given $D$, the element $A_{D}$ is uniquely defined up to adding a scalar matrix.

Proof. In this proof, $i, j, k, l, m$ are always indices from 1 to $n$.

The algebra $\mathfrak{o}_{I}^{(1)}(n)$ consists of zero-diagonal symmetric $n \times n$ matrices, which means that the elements $o^{\{i, j\}}:=E^{i, j}+E^{j, i}$, where $\{i, j\}$ are all two-element subsets of $\{1, \ldots, n\}$, form a basis of $\mathfrak{o}_{I}^{(1)}(n)$. Their commutation relations are (we assume that $i \neq j$ and $k \neq l$ ):

$$
\left[o^{\{i, j\}}, o^{\{k, l\}}\right]= \begin{cases}0 & \text { if }\{k, l\}=\{i, j\} \text { or }\{k, l\} \cap\{i, j\}=\varnothing \\ o^{\{j, k\}} & \text { for } l=i \text { and } k \neq i, j .\end{cases}
$$

Alternatively, we can say that for an arbitrary matrix $M \in \mathfrak{o}_{I}^{(1)}(n)$ and $i \neq j$,

$$
\begin{aligned}
& {\left[M, o^{\{i, j\}}\right]_{k l}=0 \quad \text { if }\{k, l\}=\{i, j\} \text { or }\{k, l\} \cap\{i, j\}=\varnothing,} \\
& {\left[M, o^{\{i, j\}}\right]_{i k}=\left[M, o^{\{i, j\}}\right]_{k i}=M_{j k} \quad \text { for } k \neq i, j,} \\
& {\left[M, o^{\{i, j\}}\right]_{k k}=0 \quad \text { for an arbitrary } k}
\end{aligned}
$$

Let $D$ be a derivation of $\mathfrak{o}_{I}^{(1)}(n)$. Let us prove that for arbitrary three pairwise distinct indices $i, j, k$,

$$
\left(D o^{\{i, j\}}\right)_{i j}+\left(D o^{\{i, k\}}\right)_{i k}+\left(D o^{\{j, k\}}\right)_{j k}=0,
$$

and that for arbitrary four pairwise distinct indices $i, j, k, l$,

$$
\begin{aligned}
& \left(D o^{\{i, j\}}\right)_{k l}=0 \\
& \left(D o^{\{i, k\}}\right)_{i l}=\left(D o^{\{j, k\}}\right)_{j l}=\left(D o^{\{i, l\}}\right)_{i k} .
\end{aligned}
$$


Since $o^{\{i, k\}}=\left[o^{\{i, j\}}, o^{\{j, k\}}\right]$, we have

$$
\begin{aligned}
\left(D o^{\{i, k\}}\right)_{i k} & =\left(D\left[o^{\{i, j\}}, o^{\{j, k\}}\right]\right)_{i k}=\left[D o^{\{i, j\}}, o^{\{j, k\}}\right]_{i k}+\left[o^{\{i, j\}}, D o^{\{j, k\}}\right]_{i k} \\
& =\left(D o^{\{i, j\}}\right)_{i j}+\left(D o^{\{k, j\}}\right)_{j k}
\end{aligned}
$$

which proves (A.1).

Note that (A.2) and (A.3) are vacuously true for $n=3$, since in this case, there exist no four pairwise distinct indices. In case of $n \geq 5$, let $m$ be an index different from all of $i, j, k, l$. Then $\left[o^{\{i, j\}}, o^{\{l, m\}}\right]=0$, which means that

$$
0=\left(D\left[o^{\{i, j\}}, o^{\{l, m\}}\right]\right)_{k m}=\left[D o^{\{i, j\}}, o^{\{l, m\}}\right]_{k m}+\left[o^{\{i, j\}}, D o^{\{l, m\}}\right]_{k m}=\left(D o^{\{i, j\}}\right)_{k l}+0,
$$

which proves (A.2). Since $o^{\{i, k\}}=\left[o^{\{i, j\}}, o^{\{j, k\}}\right]$, we have

$$
\left(D o^{\{i, k\}}\right)_{i l}=\left(D\left[o^{\{i, j\}}, o^{\{j, k\}}\right]\right)_{i l}=\left[D o^{\{i, j\}}, o^{\{j, k\}}\right]_{i l}+\left[o^{\{i, j\}}, D o^{\{j, k\}}\right]_{i l}=0+\left(D o^{\{j, k\}}\right)_{j l}
$$

which proves the first equality in (A.3). And since $o^{\{i, k\}}=\left[o^{\{i, l\}}, o^{\{k, l\}}\right]$, we have

$$
\left(D o^{\{i, k\}}\right)_{i l}=\left(D\left[o^{\{i, l\}}, o^{\{k, l\}}\right]\right)_{i l}=\left[D o^{\{i, l\}}, o^{\{k, l\}}\right]_{i l}+\left[o^{\{i, l\}}, D o^{\{k, l\}}\right]_{i l}=\left(D o^{\{i, l\}}\right)_{i k}+0
$$

which proves the second equality in (A.3).

Now consider matrix $A_{D}$ whose entries are as follows:

$$
\begin{aligned}
& \left(A_{D}\right)_{11}=0, \quad\left(A_{D}\right)_{i i}=\left(D o^{\{1, i\}}\right)_{1 i} \quad \text { for } i \neq 1, \\
& \left(A_{D}\right)_{i j}=\left(D o^{\{k, i\}}\right)_{k j} \quad \text { for } i \neq j, \text { and some } k \neq i, j .
\end{aligned}
$$

Note that (A.3) shows that $\left(A_{D}\right)_{i j}$ does not depend on the choice of $k$ and that the matrix is symmetric, i.e., $\left(A_{D}\right)_{i j}=\left(A_{D}\right)_{j i}$.

Then it follows from (A.1), (A.2) and (A.3) that $D o^{\{i, j\}}=\left[A_{D}, o^{\{i, j\}}\right]$ for an arbitrary $o^{\{i, j\}}$. Since the $o^{\{i, j\}}$ form a basis of $\mathfrak{o}_{I}^{(1)}(n)$, it means that $D$ coincides with the restriction of $\operatorname{ad}_{A_{D}}$ to $\mathfrak{o}_{I}^{(1)}(n)$. On the other hand, it is easy to check that for any matrix $A \in \mathfrak{o}_{I}(n)$, the restriction of $\operatorname{ad}_{A}$ to $\mathfrak{o}_{I}^{(1)}(n)$ is a derivation of $\mathfrak{o}_{I}^{(1)}(n)$, and that two matrices $A, A^{\prime} \in \mathfrak{o}_{I}(n)$ determine the same derivation of $\mathfrak{o}_{I}^{(1)}(n)$ if and only if $A-A^{\prime}=c 1_{n}$ for some $c \in \mathbb{K}$. This proves our claim.

\section{Acknowledgements}

We are thankful to D. Leites, who raised the problem, I. Shchepochkina, and S. Skryabin for help, and to P. Grozman, whose code SuperLie, see [15], we used in our computer experiments. Thanks are due to S. Bouarroudj, and V. Grandjean for discussions and useful comments. The first author thanks the Organising committee of the symposium "Groningen Deformation Day" (October 7, 2016, Groningen, The Netherlands), where the results of this note were delivered, for hospitality and financial support; his research was partly supported by WCMCS post-doctoral fellowship and the grant AD 065 NYUAD during his visits of NYUAD. For the possibility to perform the difficult computations of this research we are grateful to M. Al Barwani, Director of the High Performance Computing resources at New York University Abu Dhabi.

\section{References}

[1] Bahturin Y., Kochetov M., Group gradings on restricted Cartan-type Lie algebras, Pacific J. Math. 253 (2011), 289-319, arXiv:1001.0191. 
[2] Bouarroudj S., Grozman P., Lebedev A., Leites D., Derivations and central extensions of simple modular Lie algebras and superalgebras, arXiv:1307.1858.

[3] Bouarroudj S., Grozman P., Lebedev A., Leites D., Shchepochkina I., Simple vectorial Lie algebras in characteristic 2 and their superizations, arXiv:1510.07255.

[4] Bouarroudj S., Grozman P., Lebedev A., Leites D., Shchepochkina I., New simple Lie algebras in characteristic 2, Int. Math. Res. Not. 2016 (2016), 5695-5726, arXiv:1307.1551.

[5] Bouarroudj S., Grozman P., Leites D., Deforms of symmetric simple modular Lie (super)algebras, arXiv:0807.3054.

[6] Bouarroudj S., Grozman P., Leites D., Classification of finite dimensional modular Lie superalgebras with indecomposable Cartan matrix, SIGMA 5 (2009), 060, 63 pages, arXiv:0710.5149.

[7] Bouarroudj S., Lebedev A., Leites D., Shchepochkina I., Classifications of simple Lie superalgebras in characteristic 2, arXiv:1407.1695.

[8] Bouarroudj S., Lebedev A., Leites D., Shchepochkina I., Lie algebra deformations in characteristic 2, Math. Res. Lett. 22 (2015), 353-402, arXiv:1301.2781.

[9] Bouarroudj S., Lebedev A., Wagemann F., Deformations of the Lie algebra $\mathfrak{o}(5)$ in characteristics 3 and 2, Math. Notes 89 (2011), 777-791, arXiv:0909.3572.

[10] Chebochko N.G., Kuznetsov M.I., Integrable cocycles and global deformations of Lie algebra of type $G_{2}$ in characteristic 2, Comm. Algebra 45 (2017), 2969-2977.

[11] Dolotkazin A.H., Irreducible representations of a simple three-dimensional Lie algebra of characteristic $p=2$, Math. Notes 24 (1978), 588-590.

[12] Eick B., Some new simple Lie algebras in characteristic 2, J. Symbolic Comput. 45 (2010), $943-951$.

[13] Elduque A., Kochetov M., Gradings on simple Lie algebras, Mathematical Surveys and Monographs, Vol. 189, Amer. Math. Soc., Providence, RI, Atlantic Association for Research in the Mathematical Sciences (AARMS), Halifax, NS, 2013.

[14] Grishkov A., Zusmanovich P., Deformations of current Lie algebras. I. Small algebras in characteristic 2, J. Algebra 473 (2017), 513-544, arXiv:1410.3645.

[15] Grozman P., SuperLie: a Mathematica package for calculations in Lie algebras and superalgebras, available at http://www. equaonline.com/math/SuperLie.

[16] Grozman P., Leites D., Structures of $G(2)$ type and nonintegrable distributions in characteristic $p$, Lett. Math. Phys. 74 (2005), 229-262, math.RT/0509400.

[17] Helgason S., Differential geometry, Lie groups, and symmetric spaces, Pure and Applied Mathematics, Vol. 80, Academic Press, Inc., New York - London, 1978.

[18] Kochetov M., Gradings on finite-dimensional simple Lie algebras, Acta Appl. Math. 108 (2009), $101-127$.

[19] Kochetov M., Parsons N., Sadov S., Counting fine grading on matrix algebras and on classical simple Lie algebras, Internat. J. Algebra Comput. 23 (2013), 1755-1781, arXiv:1210.4589.

[20] Kostrikin A.I., The beginnings of modular Lie algebra theory, in Group Theory, Algebra, and Number Theory (Saarbrücken, 1993), de Gruyter, Berlin, 1996, 13-52.

[21] Kuznetsov M.I., Maximal tori of a general Lie algebra of Cartan type, Sb. Math. 188 (1997), 1317-1342.

[22] Lebedev A., Analogs of the orthogonal, Hamiltonian, Poisson, and contact Lie superalgebras in characteristic 2, J. Nonlinear Math. Phys. 17 (2010), suppl. 1, 217-251.

[23] Permiakov D.S., Derivations of classical Lie algebras over the field of characteristic 2, Vestnik Lobachevsky State Univ. Nizhni Novgorod Ser. Math. 1 (2005), 123-134, available at http://www.vestnik.unn.ru/en/ nomera?anum $=1455$.

[24] Richardson Jr. R.W., On the rigidity of semi-direct products of Lie algebras, Pacific J. Math. 22 (1967), 339-344.

[25] Skryabin S., Classification of Hamiltonian forms over divided power algebras, Math. USSR-Sb. 69 (1991), 121-141.

[26] Skryabin S., Toral rank one simple Lie algebras of low characteristics, J. Algebra 200 (1998), 650-700.

[27] Skryabin S., On the automorphism group schemes of Lie algebras of Witt type, Comm. Algebra 29 (2001), 4047-4077.

[28] Strade H., Simple Lie algebras over fields of positive characteristic. I. Structure theory, De Gruyter Expositions in Mathematics, Vol. 38, Walter de Gruyter \& Co., Berlin, 2004.

[29] Tyurin S.A., Classification of tori in the Zassenhaus algebra, Russian Math. (Iz. VUZ) 42 (1998), no. 2, 66-73. 